\title{
Rosmarinic Acid - Pharmaceutical and Clinical Aspects
}

Authors

Affiliation
Solomon K. S. Amoah, Louis P. Sandjo, Jadel M. Kratz, Maique W. Biavatti

Postgraduate Program in Pharmacy, Universidade Federal de Santa Catarina - UFSC, Florianópolis, SC, Brazil

\author{
Key words \\ - rosmarinic acid \\ - isolation and analysis \\ - clinical studies \\ - pharmacokinetics \\ - pharmaceutical \\ development
}

received March 5, 2015 revised Nov. 20,2015 accepted Dec. 7, 2015

\section{Bibliography}

DOI http://dx.doi.org/

10.1055/s-0035-1568274

Published online February 4, 2016

Planta Med 2016; 82: 388-406

(c) Georg Thieme Verlag KC

Stuttgart · New York .

ISSN 0032-0943

\section{Correspondence}

Maique W. Biavatti

Laboratório de Farmacognosia CIF/CCS/UFSC

Campus Universitário/Trindade 88040-900 Florianópolis, SC Brazil

Phone: + 554837213493

maique.biavatti@ufsc.br

\section{Abstract}

$\nabla$

The biosynthesis and biotechnological production of Rosmarinic acid, a phenolic ester that is widespread in the plant kingdom, has been widely investigated. This compound has shown many remarkable biological and pharmacological activities, which have led to its pharmaceutical and analytical development, as well as clinical studies, which are summarized and analyzed here for the first time. This review compiles data from the Pubmed, Scopus, Scifinder, Web Of Science, and Science Direct databases published between 1990 and 2015, restricting the search to works with the keywords "Rosmarinic acid" in the title. The initial search identified more than 800 articles; after an initial screening and removal of duplicate works, the search was further refined, resulting in approximately 300 articles that were scrutinized and comprise this review. The articles were organized to describe extraction and isolation, analytical methods, pharmaceutical development, and biological and pharmacological activities [divided into nonclinical (in vitro, in vivo) and clinical studies], pharmacokinetic studies, and stability studies.

\section{Abbreviations \\ $\nabla$ \\ 6-OHDA: 6-hydroxydopamine \\ CCL11: C-C motif chemokine 11}

\section{Introduction}

$\nabla$

The natural compound RA [(R)-(+)-rosmarinic acid, 0 Fig. 1 is a common ester derived from caffeic acid and (R)-(+)-3-(3,4-dihydroxyphenyl)lactic acid that can accumulate in high amounts in many plant species. RA is abundant in several medicinal plants of the Lamiaceae family, such as
CC: $\quad$ column chromatography

DAD: diode array detector

DPPH: 2,2-diphenyl-1-picrylhydrazyl

ELISA: enzyme-linked immunosorbent assay

ESIMS: electrospray ionization mass spectrometry

HPLC: high performance liquid chromatography

(HP)TLC: (high-performance) thin-layer chromatography

IDO: $\quad$ indoleamine 2,3-dioxygenase

IKK- $\beta$ : $\quad$ I $k$ B kinase $\beta$

LC: liquid chromatography

LPS: lipopolysaccharide

MPLC: medium performance liquid chromatography

MS: mass spectrometry

NF- $k$ B: nuclear factor-kappa B

NMR: nuclear magnetic resonance

PDA: $\quad$ photo diode array

PK: $\quad$ pharmacokinetic

PLE: $\quad$ pressurized liquid extraction

RA: rosmarinic acid

SCORAD: severity scoring of atopic dermatitis

SFE: $\quad$ supercritical fluid extraction

t-BOOH: tert-butylhydroperoxide

TEWL: $\quad$ transepidermal water loss

TNF- $\alpha$ : $\quad$ tumor necrosis factor- $\alpha$

UAE: ultrasonic-assisted extraction

UPLC: ultra performance liquid chromatography

UV-Vis: ultraviolet-visible spectroscopy

rosemary (Rosmarinus officinalis L.), spearmint (Mentha spp.), and lemon balm (Melissa officinalis L.), and also in plants used in traditional Chinese medicine, such as Perilla frutescens (L.) Britton, Salvia miltiorrhiza Bunge, and Rabdosia rubescens (Hemsl.) H. Hara. Many studies have reported the role of RA in the biological activity of these plants as well as its pharmaceutical and biotechnological 


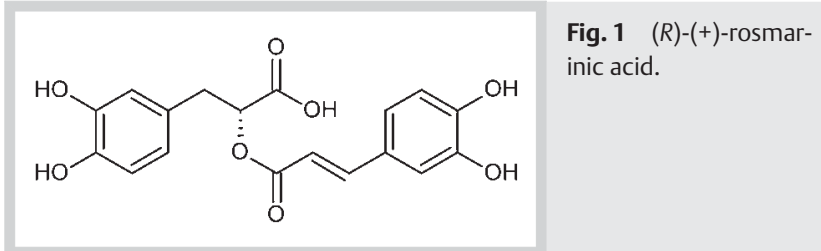

applications. Concerning its aspects in the biotechnology field, many reviews have been published in the last decade [1-6]. The present review covers predominantly analytical, pharmaceutical, and biological aspects of RA, including clinical trials.

\section{Extraction, Isolation, and Analytical Methods \\ $\nabla$}

Several extraction protocols [7-11], separation [12-14], and chromatographic methods have been developed to optimize the extraction and identification of RA $[7,12,15]$. The type of solvents, temperature, extraction time, particle size of the material to be extracted, and ratio of the solvents are some of the parameters studied [16-19]. Sometimes solvent modifiers were used, such as acids, bases, and salts, and ionic solvents have also been explored $[8,20,21]$. Moreover, various methods and techniques used to extract polyphenols from plant materials have ended up obtaining RA as one of the principal components [22-26]. Table 1 shows a summary of selected techniques for RA extraction. Versatile extraction of RA requires a good choice of protic solvents, temperature, and sufficient time to avoid degradation in analytical studies or optimization processes. Response surface methodology has also been used to optimize the isolation of RA [27-31].

Along with conventional extraction methods, for instance hydrodistillation, decoction, and maceration, other methods, such as PLE [32], UAE [29,33,34], SFE [35], microwave-assisted extraction [23,36-39], accelerated solvent extraction [40], and enzyme-assisted extraction [41,42], have also been employed to extract polyphenols, of which RA was identified as one of the major compounds. Nanofiltration [43-45] and solid-phase extraction $[46,47]$ are examples of techniques used to improve the amount of RA extracted from plant materials.

Interestingly, in a study that used infusion, decoction, microwave, and ultrasonic methods to optimize the extraction of phenolic compounds from Plectranthus spp., the highest amount of RA was found in the decoction extract of Plectranthus ecklonii Benth. (Lamiaceae) (293 $\mu \mathrm{M})$ [48]. Cabana and collaborators [49] prepared hydrolate, decoction, and ethanolic extracts from Satureja parvifolia (Phil.) Epling (Lamiaceae) and demonstrated that the hydrolate extract presented the highest amount of RA.

In some studies, conventional soxhlet extraction was compared with other methods. Dracocephalum moldavica L. (Lamiaceae) was extracted using a soxhlet apparatus with several solvents, of which $n$-butanol extract presented the highest RA content (114.54 $\pm 24.70 \mathrm{mg} / \mathrm{g}$ dry extract) [50]. Soxhlet extraction was compared with the maceration method on unripe, half ripe, and full ripe Cuminum cyminum L. (Apiaceae) seeds [51], demonstrating that the maceration method produced a higher RA content than the soxhlet extraction. By contrast, a comparison between soxhlet and SFE of Satureja hortensis L. showed that soxhlet extraction with ethanol provided the highest RA content [52].

With SFE of $R$. officinalis (rosemary), comparison of $\mathrm{CO}_{2}$ with dimethyl ether demonstrated that dimethyl ether provided the highest RA yield [53]. The same plant leaves treated with supercritical fluid- $\mathrm{CO}_{2}$ at 5000 psi and 40,60 , and $80^{\circ} \mathrm{C}$ showed that extraction at $60^{\circ} \mathrm{C}$ furnished the highest amount of RA [54]. However, comparative studies on rosemary leaves using SFE, the hydrodistillation method, and solvent extraction with different solvents showed that the RA content was highest in the solvent extraction with ethanol [55]. Again in rosemary leaves, PLE using water and ethanol as solvents, compared to SFE using neat $\mathrm{CO}_{2}$ and supercritical $\mathrm{CO}_{2}$ modified with ethanol, showed that PLE using ethanol at temperature $\left(150^{\circ} \mathrm{C}\right)$ produced the highest RA yield [56].

From these comparative studies, it cannot be concluded that there is an ideal solvent or method to obtain RA, as it depends on the combination of chosen parameters.

To provide one-step isolation and purification of RA from S. miltiorrhiza, an online adsorption chromatography-countercurrent chromatography was carried out after UAE, adsorbing the plant extract on the resin and immediately submitting it to high-speed countercurrent chromatography (HSCCC), which led to the isolation of RA with a purity of about $88 \%$ [12].

A template-assisted process using the supramolecular technique has also been used to extract RA from $P$. frutescens leaves. A P. frutescens extract, separated by supramolecular formation using flavocommelin and magnesium acetate aqueous solution followed by solvent extraction (ethyl acetate and water), yielded RA with $62.9 \pm 4.5 \%$ purity [58].

Analytical techniques used to determine RA include capillary electrophoresis [59], electromechanical [60], spectroscopical [61-64], and chromatographic methods [65-71].

Some unusual electrochemical techniques have been developed, such as that reported by Franzoi et al. [72], which uses a biosensor based on laccase from the fungus Aspergillus oryzae and the ionic liquid 1-n-butyl-3-methylimidazolium hexafluorophosphate (BMIPF6) to determine RA in M. officinalis by square wave voltammetry. Additionally, a synthetic heterodinuclear Fe(III)Zn (II) complex, a biomimetic complex of purple acid phosphatases, has also shown to be effective in determining RA in plant extracts [60]. The amount of RA determined by these techniques was in accordance with capillary electrophoresis data. Another electrochemical technique using dual-channel coulometric detection coupled with liquid chromatography has also been used to quantify RA in Prunella vulgaris L. (Lamiaceae), animal feed, and pig plasma, with minimum preseparation steps, offering good selectivity and sensitivity [69].

Standard chromatographic techniques are the most frequent form of analysis used in the determination of RA. These can be grouped into TLC densitometry or HPTLC, and LC methods. TLC/ HPTLC methods are the most accessible ones, but provide variable quantitative results since some methods possess methodological weakness. For example, integrated in the development process of a new method, a validation step is mandatory to demonstrate its reliability and the suitability. These methods used diverse stationary phases, such as normal silica gel [65, 73-77] and chemically modified silica gel with amino, cyano, or octadecyl groups $[70,78,79]$. Using densitometry, Janicsak et al. [76] showed the RA content in some species of Lamiaceae ranging from 0.01 to $9.30 \mathrm{mg} / \mathrm{g}$.

Regarding LC methods, they have been most widely used for the separation, detection, and quantification of RA in plant extracts, providing a wide range of concentrations of RA in several Lamiaceae species. UV-Vis or DAD/PDA are the most widely used detectors $[66,67,68,75,80]$, followed by an MS detector $[67,71,75,81]$. 
Table 1 Summary of extraction and isolation studies for rosmarinic acid.

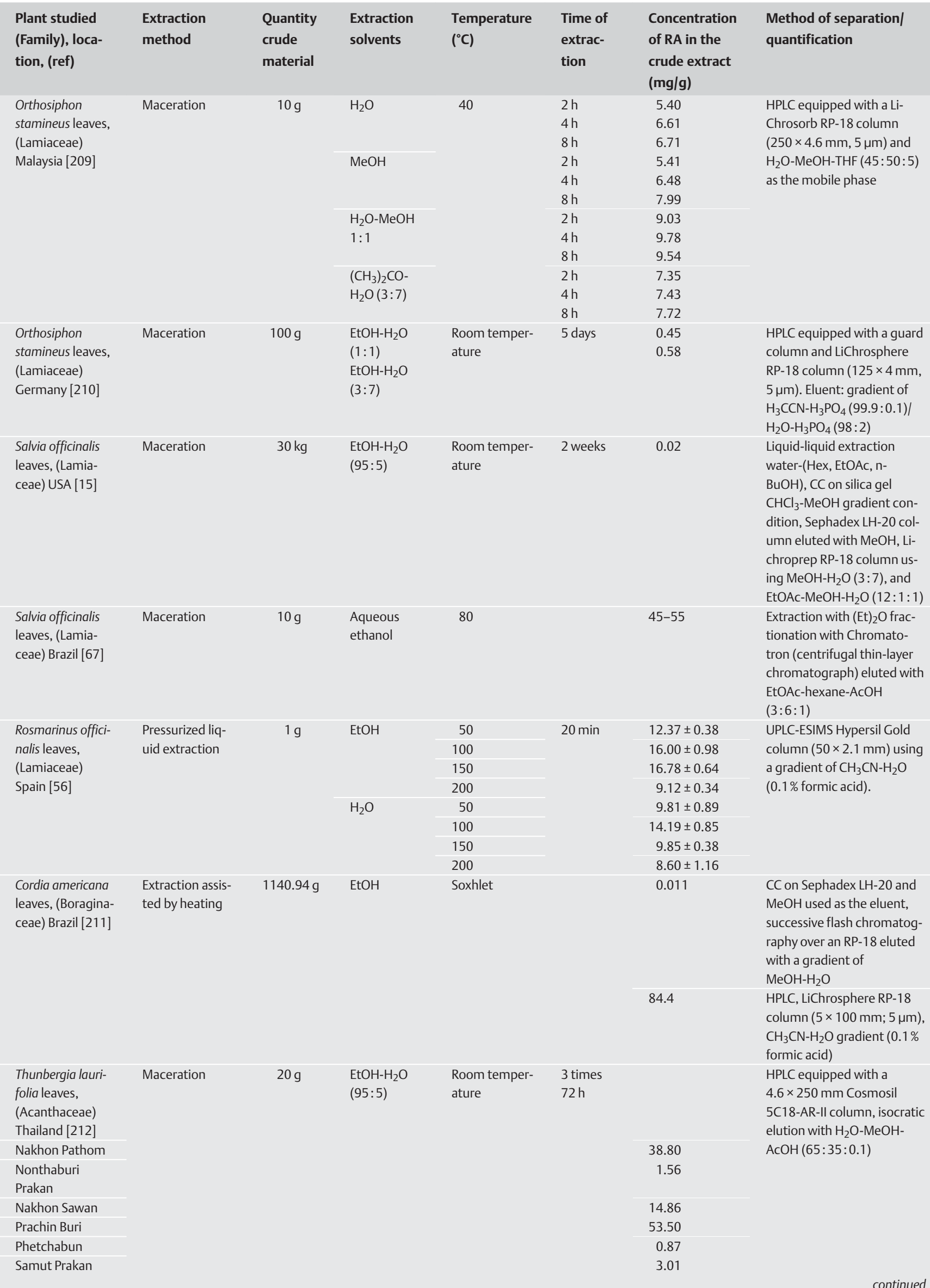


Table 1 Continued

\begin{tabular}{|c|c|c|c|c|c|c|c|}
\hline $\begin{array}{l}\text { Plant studied } \\
\text { (Family), loca- } \\
\text { tion, (ref) }\end{array}$ & $\begin{array}{l}\text { Extraction } \\
\text { method }\end{array}$ & $\begin{array}{l}\text { Quantity } \\
\text { crude } \\
\text { material }\end{array}$ & $\begin{array}{l}\text { Extraction } \\
\text { solvents }\end{array}$ & $\begin{array}{l}\text { Temperature } \\
\left({ }^{\circ} \mathrm{C}\right)\end{array}$ & $\begin{array}{l}\text { Time of } \\
\text { extrac- } \\
\text { tion }\end{array}$ & $\begin{array}{l}\text { Concentration } \\
\text { of RA in the } \\
\text { crude extract } \\
(\mathrm{mg} / \mathrm{g})\end{array}$ & $\begin{array}{l}\text { Method of separation/ } \\
\text { quantification }\end{array}$ \\
\hline $\begin{array}{l}\text { Mentha piperita } \\
\text { leaves, (Lamia- } \\
\text { ceae) Iraq [16] }\end{array}$ & \multirow[t]{3}{*}{$\begin{array}{l}\text { Maceration } \\
\text { assisted by } \\
\text { ultrasound }\end{array}$} & \multirow[t]{3}{*}{$1 \mathrm{~g}$} & \multirow[t]{3}{*}{$\begin{array}{l}\mathrm{EtOH}-\mathrm{H}_{2} \mathrm{O} \\
(4: 1)\end{array}$} & \multirow[t]{3}{*}{40} & \multirow[t]{3}{*}{$1 \mathrm{~h}$} & 1.43 & \multirow{3}{*}{$\begin{array}{l}\mathrm{HPLC} \text { instrument, } \mathrm{C} 18 \mathrm{col}- \\
\text { umn }(4.6 \times 250 \mathrm{~mm}, 5 \mathrm{~mm}) \text {, } \\
\text { isocratic } \mathrm{MeOH}-\mathrm{H}_{2} \mathrm{O}+0.1 \% \\
\mathrm{AcOH}(4: 1)\end{array}$} \\
\hline $\begin{array}{l}\text { Mentha longifolia } \\
\text { leaves, (Lamia- } \\
\text { ceae) Iraq }\end{array}$ & & & & & & 2.08 & \\
\hline $\begin{array}{l}\text { Osimum basilicum } \\
\text { leaves, (Lamia- } \\
\text { ceae) Iraq }\end{array}$ & & & & & & 3.06 & \\
\hline $\begin{array}{l}\text { Maranta depressa } \\
\text { leaves, (Maranta- } \\
\text { ceae) Germany } \\
{[213]}\end{array}$ & \multirow[t]{6}{*}{$\begin{array}{l}\text { Extraction } \\
\text { assisted by } \\
\text { sonication }\end{array}$} & \multirow[t]{6}{*}{$50 \mathrm{mg}$} & \multirow[t]{6}{*}{ EtOH 70\% } & \multirow[t]{6}{*}{70} & \multirow[t]{6}{*}{$10 \mathrm{~min}$} & 12.6 & \multirow{6}{*}{$\begin{array}{l}\text { HPLC on a Hypersil ODS col- } \\
\text { umn }(5 \mathrm{~mm}, 280 \mathrm{~mm} \\
\times 40 \mathrm{~mm}) \text { and } 45 \% \mathrm{MeOH} / \\
0.01 \% \mathrm{H}_{3} \mathrm{PO}_{4} \text { used as the } \\
\text { eluent }\end{array}$} \\
\hline $\begin{array}{l}\text { Maranta leuco- } \\
\text { neura “Fascina- } \\
\text { tor” leaves, } \\
\text { (Marantaceae) } \\
\text { Germany }\end{array}$ & & & & & & 8.7 & \\
\hline $\begin{array}{l}\text { Maranta leuco- } \\
\text { neura var. ker- } \\
\text { choviana leaves, } \\
\text { (Marantaceae) } \\
\text { Germany }\end{array}$ & & & & & & 8.1 & \\
\hline $\begin{array}{l}\text { Maranta leuco- } \\
\text { neura var. mas- } \\
\text { sangeana leaves, } \\
\text { (Marantaceae) } \\
\text { Germany }\end{array}$ & & & & & & 7.8 & \\
\hline $\begin{array}{l}\text { Canna edulis } \\
\text { leaves, (Canna- } \\
\text { ceae) Germany }\end{array}$ & & & & & & 37.2 & \\
\hline $\begin{array}{l}\text { Canna indica } \\
\text { leaves, (Canna- } \\
\text { ceae) Germany }\end{array}$ & & & & & & 50.7 & \\
\hline $\begin{array}{l}\text { Perilla frutescens } \\
\text { leaves, (Lamia- } \\
\text { ceae) Japan [18] }\end{array}$ & Maceration & $650 \mathrm{~g}$ & $\mathrm{MeOH}$ & $\begin{array}{l}\text { Room temper- } \\
\text { ature }\end{array}$ & & 1.9 & $\begin{array}{l}\text { Acidification, amberlite col- } \\
\text { umn eluted with acidified } \\
\mathrm{H}_{2} \mathrm{O} \text { and } \mathrm{MeOH} \text {, droplet } \\
\text { countercurrent chromatog- } \\
\text { raphy eluted with } \mathrm{CHCl}_{3}-n- \\
\mathrm{BuOH}-\mathrm{MeOH}-\mathrm{H}_{2} \mathrm{O} \\
(9: 2: 12: 8) \text { and } \mathrm{CC} \text { (Diaion } \\
\mathrm{HP} 2 \mathrm{OAG} \text { ) eluted with } \mathrm{H}_{2} \mathrm{O}- \\
\mathrm{MeOH} \text { in gradient conditions }\end{array}$ \\
\hline $\begin{array}{l}\text { Melissa officinalis } \\
\text { leaves, (Lamia- } \\
\text { ceae) Slovak [19] }\end{array}$ & Maceration & & $\begin{array}{l}i \mathrm{PrOH}-\mathrm{H}_{2} \mathrm{O} \\
(39: 61)\end{array}$ & 66 & $1 \mathrm{~h}$ & $72.6 \pm 3.1$ & $\begin{array}{l}\text { HPLC analysis RP18, } \\
250 \times 4 \mathrm{~mm}, 5 \mu \mathrm{m} \text {, gradient } \\
\text { condition of } \mathrm{CH}_{3} \mathrm{CN}-\mathrm{H}_{2} \mathrm{O} \text {, } \\
0.1 \% \mathrm{CF}_{3} \mathrm{CO}_{2} \mathrm{H}\end{array}$ \\
\hline $\begin{array}{l}\text { Anthurium versi- } \\
\text { color leaves, } \\
\text { (Araceae) } \\
\text { Ecuador [214] }\end{array}$ & Maceration & $195 \mathrm{~g}$ & $\mathrm{MeOH}$ & $\begin{array}{l}\text { Room temper- } \\
\text { ature }\end{array}$ & & 0.031 & $\begin{array}{l}\text { Chromatography on Sepha- } \\
\text { dex LH-20, RP-HPLC on a C18 } \\
\text { Bondapack column }(30 \mathrm{~cm} \\
\times 7.8 \mathrm{~mm} \text { ) eluent isocratic } \\
\mathrm{MeOH}-\mathrm{H}_{2} \mathrm{O}(1: 1)\end{array}$ \\
\hline $\begin{array}{l}\text { Tournefortia sar- } \\
\text { mentosa (Boragi- } \\
\text { naceae) Taiwan } \\
{[215]}\end{array}$ & Maceration & $20 \mathrm{~kg}$ & $\begin{array}{l}\text { EtOH- } \mathrm{H}_{2} \mathrm{O} \\
(85: 15)\end{array}$ & 50 & & 0.625 & $\begin{array}{l}\text { Diaion } \mathrm{HP}-20 \text { column chro- } \\
\text { matography using a gradient } \\
\text { of } \mathrm{MeOH}-\mathrm{H}_{2} \mathrm{O} \text { and Sephadex } \\
\mathrm{LH}-20 \text { using } \mathrm{MeOH} 80 \%\end{array}$ \\
\hline $\begin{array}{l}\text { Salvia officinalis } \\
\text { (Lamiaceae) } \\
\text { New Zealand } \\
{[216]}\end{array}$ & Maceration & $50 \mathrm{~g}$ & $\begin{array}{l}\mathrm{Me}_{2} \mathrm{CO}-\mathrm{H}_{2} \mathrm{O} \\
(7: 3)\end{array}$ & $\begin{array}{l}\text { Room temper- } \\
\text { ature }\end{array}$ & & 48.65 & $\begin{array}{l}\text { Diaion } \mathrm{HP}-20 \text { column, eluent } \\
\mathrm{H}_{2} \mathrm{O} \text { then } \mathrm{MeOH} \text {, Sephadex } \\
\mathrm{LH}-20 \text { column } \mathrm{MeOH}-\mathrm{H}_{2} \mathrm{O} \\
\text { ( } 7: 3) \text {, } \mathrm{HPLC} \text { equipped with } \\
\text { an } \mathrm{RP}-18 \text { column elution } \\
\text { gradient of } \mathrm{CH}_{3} \mathrm{CN}-\mathrm{H}_{2} \mathrm{O}(2 \% \\
\mathrm{AcOH} \text { ) }\end{array}$ \\
\hline
\end{tabular}


Table 1 Continued

\begin{tabular}{|c|c|c|c|c|c|c|c|}
\hline $\begin{array}{l}\text { Plant studied } \\
\text { (Family), loca- } \\
\text { tion, (ref) }\end{array}$ & $\begin{array}{l}\text { Extraction } \\
\text { method }\end{array}$ & $\begin{array}{l}\text { Quantity } \\
\text { crude } \\
\text { material }\end{array}$ & $\begin{array}{l}\text { Extraction } \\
\text { solvents }\end{array}$ & $\begin{array}{l}\text { Temperature } \\
\left({ }^{\circ} \mathrm{C}\right)\end{array}$ & $\begin{array}{l}\text { Time of } \\
\text { extrac- } \\
\text { tion }\end{array}$ & $\begin{array}{l}\text { Concentration } \\
\text { of RA in the } \\
\text { crude extract } \\
(\mathrm{mg} / \mathrm{g})\end{array}$ & $\begin{array}{l}\text { Method of separation/ } \\
\text { quantification }\end{array}$ \\
\hline $\begin{array}{l}\text { Sanicula europaea } \\
\text { aerial parts, } \\
\text { (Apiaceae) Turkey } \\
\text { [217] }\end{array}$ & Maceration & $940 \mathrm{~g}$ & $\begin{array}{l}\mathrm{EtOH}-\mathrm{H}_{2} \mathrm{O} \\
(1: 1)\end{array}$ & 65 & $1 \mathrm{~h}$ & 0.26 & $\begin{array}{l}\text { Column chromatography on } \\
\text { silica gel } 60 \mathrm{G} \text { mixed with sil- } \\
\text { ica gel } 60 \mathrm{HF} 254 \text { and elution } \\
\text { performed with } \mathrm{CHCl}_{3-} \\
\mathrm{MeOH}-\mathrm{H}_{2} \mathrm{O}(61: 32: 7)\end{array}$ \\
\hline $\begin{array}{l}\text { Perilla frutescens } \\
\text { seeds, (Lamia- } \\
\text { ceae) Japan [218] }\end{array}$ & $\begin{array}{l}\text { Extraction assis- } \\
\text { ted by heating }\end{array}$ & $800 \mathrm{~g}$ & $\mathrm{EtOH}$ & Reflux & $2 \mathrm{~h}$ & 0.024 & $\begin{array}{l}\text { Liquid-liquid extraction Hex, } \\
\text { EtOAc- } \mathrm{H}_{2} \mathrm{O} \text {, EtOAc. Fraction } \\
\text { purified by CC silica gel, Se- } \\
\text { phadex LH-20 and HPLC } \\
\text { equipped with an ODS col- } \\
\text { umn, } \mathrm{MeOH}-\mathrm{H}_{2} \mathrm{O}(1: 1) \text { used } \\
\text { as the eluent }\end{array}$ \\
\hline $\begin{array}{l}\text { Nepeta cadmea } \\
\text { aerial parts, } \\
\text { (Lamiaceae) } \\
\text { Turkey [14] }\end{array}$ & Maceration & $1.25 \mathrm{~kg}$ & $\mathrm{MeOH}$ & $\begin{array}{l}\text { Room temper- } \\
\text { ature }\end{array}$ & 30 days & 0.024 & $\begin{array}{l}\text { Liquid-liquid extraction Hex- } \\
\mathrm{MeOH}, \mathrm{H}_{2} \mathrm{O}-(\mathrm{EtOAc}, n- \\
\mathrm{BuOH}) . \\
\text { rified with } \mathrm{CC} \text { on Dianion HP- } \\
20 \mathrm{MeOH}-\mathrm{H}_{2} \mathrm{O} \text { Gradient. CC } \\
\text { on silica gel eluted with } \\
\mathrm{CHCl}_{3}-\mathrm{MeOH} \text { and } \mathrm{HPLC} \\
\text { equipped with a C- } 18 \text { col- } \\
\text { umn eluted with a gradient } \\
\text { of } \mathrm{MeOH}-\mathrm{H}_{2} \mathrm{O}\end{array}$ \\
\hline $\begin{array}{l}\text { Pulsatilla koreana } \\
\text { roots, (Ranuncu- } \\
\text { laceae) South } \\
\text { Korea [219] }\end{array}$ & Maceration & $1.2 \mathrm{~kg}$ & $\begin{array}{l}\mathrm{MeOH} \text { and } \\
\mathrm{CH}_{2} \mathrm{Cl}_{2}\end{array}$ & $\begin{array}{l}\text { Room temper- } \\
\text { ature }\end{array}$ & & 0.017 & $\begin{array}{l}\text { Vacuum flash chromatogra- } \\
\text { phy on } \mathrm{RP}-18 \text { using } \mathrm{MeOH} \\
\text { and } \mathrm{H}_{2} \mathrm{O} \text { as the eluent in gra- } \\
\text { dient condition. Further pu- } \\
\text { rification took place with re- } \\
\text { versed-phase } \mathrm{HPLC}(\mathrm{YM}- \\
\text { C-ODS column; } \mathrm{H}_{2} \mathrm{O}-\mathrm{MeOH} \\
\text { gradient) }\end{array}$ \\
\hline $\begin{array}{l}\text { Hyssopus cuspida- } \\
\text { tus whole plant, } \\
\text { (Lamiaceae) } \\
\text { China [220] }\end{array}$ & $\begin{array}{l}\text { Maceration } \\
\text { assisted by } \\
\text { ultrasonication }\end{array}$ & $1.5 \mathrm{~kg}$ & $\mathrm{EtOH}$ & $20-30$ & $30 \mathrm{~min}$ & 0.01 & $\begin{array}{l}\text { Diaion } \mathrm{HP} \text { - } 20 \text { resin column } \\
\text { eluted with a gradient of } \\
\mathrm{MeOH}-\mathrm{H}_{2} \mathrm{O} \text { acetone, silica } \\
\text { gel CC eluted with } \mathrm{CHCl}_{3} \text { - } \\
\mathrm{MeOH}-\mathrm{H}_{2} \mathrm{O} \text { reversed-phase } \\
\mathrm{HPLC} \text { with mobile phase } \\
\mathrm{MeOH}-\mathrm{H}_{2} \mathrm{O}(2: 6) \text { and re- } \\
\text { versed-phase } \mathrm{HPLC} \\
\mathrm{CH}_{3} \mathrm{CN}-\mathrm{H}_{2} \mathrm{O}(1: 9) \text { as the } \\
\text { mobile phase }\end{array}$ \\
\hline $\begin{array}{l}\text { Baccharis chilco } \\
\text { aerial parts, } \\
\text { (Asteraceae) } \\
\text { Colombia [10] }\end{array}$ & $\begin{array}{l}\text { Extraction assis- } \\
\text { ted by heating }\end{array}$ & $446 \mathrm{~g}$ & $\begin{array}{l}\mathrm{CH}_{2} \mathrm{Cl}_{2} \text { and } \\
\mathrm{EtOH}\end{array}$ & Reflux & $2 \mathrm{~h}$ & 0.045 & $\begin{array}{l}\mathrm{CC} \text { on silica gel with } \mathrm{CH}_{2} \mathrm{Cl}_{2}- \\
\text { EtOH used as the eluent. } \mathrm{H}_{2} \mathrm{O} \\
\text { (alkaline)-EtOAc separation } \\
\text { and } \mathrm{HPLC} \text { purification of the } \\
\text { organic phase using isocratic } \\
\text { the condition of } \mathrm{MeOH}-\mathrm{H}_{2} \mathrm{O} \\
(2: 3)\end{array}$ \\
\hline $\begin{array}{l}\text { Helicteres isora } \\
\text { fruits, (Sterculia- } \\
\text { ceae) Indonesia } \\
{[221]}\end{array}$ & $\begin{array}{l}\text { Extraction assis- } \\
\text { ted by heating }\end{array}$ & $4 \mathrm{~kg}$ & $\mathrm{MeOH}$ & Reflux 60 & $7 \mathrm{~h}$ & 0.078 & $\begin{array}{l}\text { Liquid-liquid extraction } \\
\mathrm{CHCl}_{3}-\mathrm{MeOH}-\mathrm{H}_{2} \mathrm{O}(5: 3: 8) \\
\text { and then with EtOAc and } n \text { - } \\
\text { BuOH. } \mathrm{CC} \text { on Sephadex } \mathrm{LH} \text { - } \\
20 \text { using } \mathrm{MeOH}-\mathrm{H}_{2} \mathrm{O}(2: 1) \text { as } \\
\text { th eluent. A second purifica- } \\
\text { tion on Sephadex } \mathrm{LH}-20 \\
\text { eluted with } \mathrm{MeOH}-\mathrm{H}_{2} \mathrm{O} \\
(2: 1) \text {, followed by successive } \\
\text { purification on an MPLC silica } \\
\text { gel column and an RP-18 } \\
\text { column eluted with EtOAc- } \\
\text { MeOH- } \mathrm{H}_{2} \mathrm{O}(60: 25: 15) \text { and } \\
\mathrm{H}_{2} \mathrm{O}-\mathrm{MeOH}(3: 1) \text {, respec- } \\
\text { tively }\end{array}$ \\
\hline
\end{tabular}


Table 1 Continued

\begin{tabular}{|c|c|c|c|c|c|c|c|}
\hline $\begin{array}{l}\text { Plant studied } \\
\text { (Family), loca- } \\
\text { tion, (ref) }\end{array}$ & $\begin{array}{l}\text { Extraction } \\
\text { method }\end{array}$ & $\begin{array}{l}\text { Quantity } \\
\text { crude } \\
\text { material }\end{array}$ & $\begin{array}{l}\text { Extraction } \\
\text { solvents }\end{array}$ & $\begin{array}{l}\text { Temperature } \\
\left({ }^{\circ} \mathrm{C}\right)\end{array}$ & $\begin{array}{l}\text { Time of } \\
\text { extrac- } \\
\text { tion }\end{array}$ & $\begin{array}{l}\text { Concentration } \\
\text { of } R A \text { in the } \\
\text { crude extract } \\
(\mathrm{mg} / \mathrm{g})\end{array}$ & $\begin{array}{l}\text { Method of separation/ } \\
\text { quantification }\end{array}$ \\
\hline $\begin{array}{l}\text { Hedyotis scandens } \\
\text { (Rubiaceae) [222] }\end{array}$ & Percolation & $9 \mathrm{~kg}$ & EtOH 95\% & $\begin{array}{l}\text { Room temper- } \\
\text { ature }\end{array}$ & & 0.006 & $\begin{array}{l}\text { Liquid-liquid extraction } \mathrm{H}_{2} \mathrm{O} \text {, } \\
\text { petroleum ether, EtOAc, and } \\
n \text {-BuOH. CC on silica gel } \\
\text { eluted with a gradient of } \\
\mathrm{CHCl}_{3}-\mathrm{MeOH} \text {. Further } \mathrm{CC} \text { on } \\
\text { silica gel eluted with a gra- } \\
\text { dient of } \mathrm{CHCl}_{3}-\mathrm{MeOH}\end{array}$ \\
\hline $\begin{array}{l}\text { Melissa officinalis } \\
\text { leaves, (Lamia- } \\
\text { ceae) [223] }\end{array}$ & $\begin{array}{l}\text { Extraction assis- } \\
\text { ted by stirring }\end{array}$ & $100 \mathrm{~g}$ & $\begin{array}{l}\text { Acidified } \\
\mathrm{H}_{2} \mathrm{O}\end{array}$ & $80-100$ & $45 \mathrm{~min}$ & 13 & $\begin{array}{l}\text { Liquid-liquid extraction with } \\
\mathrm{Et}_{2} \mathrm{O} \text {, Sephadex LH- } 20 \text { using } \\
\mathrm{MeOH}-\mathrm{H}_{2} \mathrm{O}(7: 3) \text {. Recrystal- } \\
\text { lization at } 4{ }^{\circ} \mathrm{C}\end{array}$ \\
\hline $\begin{array}{l}\text { Zostera noltii } \\
\text { leaves, (Zostera- } \\
\text { ceae) France } \\
{[224]}\end{array}$ & $\begin{array}{l}\text { Extraction assis- } \\
\text { ted by heating }\end{array}$ & $500 \mathrm{~g}$ & $\mathrm{MeOH}$ & Reflux & & 14.4 & $\begin{array}{l}\text { Liquid-liquid extraction } \\
\mathrm{CHCl}_{3} \text {, EtOAc and } \mathrm{H}_{2} \mathrm{O} \text {, then } \\
\text { flash chromatography on a } \\
\text { silica gel using EtOAc-MeOH } \\
(95: 5) \text { as the eluent }\end{array}$ \\
\hline Collection July & & & & & & $8.10 \pm 0.11$ & HPLC equipped with a Hy- \\
\hline Collection & & & & & & $2.17 \pm 0.27$ & persil GOLD C8 column \\
\hline December & & & & & & & $\begin{array}{l}\text { ( } 5 \mu \mathrm{m} \text { particle size, } \\
250 \times 4.6 \mathrm{~mm}) \text { and }\end{array}$ \\
\hline $\begin{array}{l}\text { Collection } \\
\text { January }\end{array}$ & & & & & & $8.65 \pm 0.11$ & $\begin{array}{l}250 \times 4.6 \mathrm{~mm}) \text { and } \\
\mathrm{MeOH}-\mathrm{H}_{2} \mathrm{O}(0.1 \% \text { TFA }) \text { used } \\
\text { as the eluent in gradient } \\
\text { conditions }\end{array}$ \\
\hline
\end{tabular}

The majority of the developed methods use UV-Vis or DAD/PDA detectors for the determination of RA in plants and extracts, while an MS detector is preferable for determination of RA in formulations and pharmacokinetic studies. Bandoniene et al. [80] used single ion monitoring mode at $m / z 383[\mathrm{M}+\mathrm{Na}$ ] to quantify RA in several Salvia spp. and Borago officinalis L. (Boraginaceae), while Berhow et al. [75] used $m / z 359$, corresponding to the [M $\mathrm{H}]^{-}$mass ion of RA, to quantify RA in Ocimum canum Sims (Lamiaceae). Moreover, LC/tandem mass spectrometry has also been exploited to determine RA and some other components in beagle dog plasma [82] during pharmacokinetic studies. Here, the deprotonated molecule $[\mathrm{M}-\mathrm{H}]^{-}$was also used, since the sensitivity in the negative mode was higher than that in the positive mode. The limit of detection was found to be $1.0 \mathrm{ng} / \mathrm{mL}$. Meanwhile, an HPLC-DAD-DPPH method has been used for online detection of the radical scavenging activity of RA [80]. After HPLC separation and UV detection at $280 \mathrm{~nm}$, the analytes reacted post-column with the DPPH at a concentration of $50 \mathrm{mg} / \mathrm{mL}$ in methanol. Trute and Nahrstedt [83] developed a laborious two-step derivatization gas chromatography method to analyze RA enantiomers.

Regarding spectroscopic methods, a spectrophotometric analysis developed by Oztürk et al. [61], based on the complexation of RA with zirconium ions, was used for the quantification of RA in 11 Salvia species. Infrared spectroscopy has also been used to quantify RA in the Lamiaceae family [62,64]. While Stehfest et al. [64] used the first derivative of the spectral region for quantification purposes, Saltas et al. [62] used the second derivative of the spectral region $1344-806 \mathrm{~cm}^{-1}$.

NMR spectroscopy has also been used to identify and quantify RA in methanol and ethanol extracts of plant species of the Lamiaceae family $[63,84]$, based on variable-temperature two-dimensional ${ }^{1} \mathrm{H}^{-1} \mathrm{H}$ double quantum filter correlation spectroscopy (DQF-COSY), ${ }^{1} \mathrm{H}^{-13} \mathrm{C}$ heteronuclear multiple-quantum coherence
(HMQC), and ${ }^{1} \mathrm{H}^{13} \mathrm{C}$ heteronuclear multiple-bond correlation (HMBC) gradient NMR spectroscopy.

\section{Pharmacological and Biological Activities}

$\nabla$

The pharmacological and biological activities of RA and RA-containing plant extracts have received considerable attention. Many scientific reports and patents have explored these properties, ranging in complexity from in vitro to clinical studies.

The exceptional antioxidant activity of RA, in parallel with the effects on cell signalling pathways and gene expression, contributes to the majority of the biological properties and technological applications covered by this review [28,85-91]. As a result, RA antioxidant and pro-oxidant profiles $[92,93]$ have been comprehensively studied, through multiple assays [93-101]. The four phenolic hydrogens underwrite its ability to modulate free radical scavenging, in combination with two catechol moieties that provide the suitable polarity for RA to penetrate the lipid bilayers and protect them against oxidation without disturbing their structure $[94,96]$. Electrochemical investigations have revealed that the first oxidation step is associated with the caffeic acid moiety, whereas the second oxidation step corresponds to the oxidation of the 3,4-dihydroxyphenyl lactic acid residue [94]. Due to the combination of these structural features, the antioxidant potential of RA is higher than that of the other hydroxycinnamic acid derivatives [96,102-104].

Besides the antioxidant potential, RA has been reported to present a multitude of pharmacological and biological activities. An in-depth description of each would be beyond the scope of this review, but a compilation and analysis of these reports is presented below (studies reporting solely extracts results were not 
included, unless otherwise stated). It is divided into three sections: in vitro, in vivo, and clinical studies.

\section{In vitro studies}

RA exhibits a wide collection of biological activities in vitro, including antioxidant, anti-inflammatory, antimutagenic, antigenotoxic, cytotoxic, antimetastatic, antiangiogenic, neuroprotective, antimicrobial, immunomodulatory, melanogenic, and antivenom effects. The array of active concentrations reported in the studies covers different magnitudes, which raises concerns about specificity. Additionally, active concentrations in the milimolar, or even high micromolar range, are unlikely to translate into clinical effects due to limitations related to plasma levels and biodistribution in humans (see section "Pharmacokinetics in $\mathrm{Hu}-$ mans"). Table 2 provides a non-exhaustive list of in vitro findings obtained with RA.

Studies that focus on the technological application of RA have reported that this phenolic acid is a good bioprotective agent against fungal infections in crops [105], and a good candidate for partial monolignol substitution via bioengineering approaches, aiming at more efficient utilization of plant fibers for biofuels or livestock production [106].

Pharmacokinetic studies of RA using in vitro models have also been conducted by some research groups. Qiang et al. compared the permeability and phase II biotransformation of RA as a pure compound and also in herbal extracts using human intestinal epithelial Caco- 2 cell monolayers. The apparent permeability coefficient $\left(\mathrm{P}_{\text {app }}\right)$ obtained for RA was $0.2 \times 10^{-6} \mathrm{~cm} / \mathrm{s}$, indicating a low permeability profile [107]. In agreement, Konishi and Kobayashi reported that in vitro intestinal absorption of RA was rather low. The majority of RA loaded on the apical side of Caco-2 cells was not transported, and the permeation occurred mainly via paracellular diffusion [108]. Moreover, RA appeared to be unsusceptible to chemical and enzymatic hydrolysis in Caco-2 cells and gastrointestinal models $[108,109]$. Conversely, the addition of Lactobacillus johnsonii cells to the rosemary extract resulted in a substantial hydrolysis of RA [109]. Taken together, these results suggest that RA is further metabolized and degraded by gut microflora, in accordance with in vivo studies (see "Pharmacokinetics in Animals" section). RA metabolites are then efficiently absorbed and distributed by the monocarboxylic acid transporter within the body [108]. Qiang et al. demonstrated that RA permeability significantly increased after treatment with $\beta$-glucuronidase/sulfatase [107], and Falé et al. demonstrated that the presence of benzoic acid, a substrate of monocarboxylic acid transporter, or flavonoids (i.e., luteolin and apigenin) can decrease RA permeation [110], supporting the involvement of membrane transporters in the uptake of RA.

The interaction of RA with other pharmaceutical relevant transporters and enzymes has been studied in vitro. Lin et al. reported RA inhibits UDP-glucuronosyltransferase activity [111], and Li et al. showed that RA could decrease the transcription and expression of P-glycoprotein, promoting the reversal of multidrug resistance phenomena [112].

\section{In vivo studies}

By compiling the in vivo studies, it was found that numerous biological studies corroborate the in vitro hypothesis. A significant prevalence of anti-inflammatory, antitumoral, and tissue damage prevention studies was also found. These reports highlight, once again, antioxidant and radical scavenging activities as key factors in the in vivo outcomes reported for RA.
The search for antitumoral compounds also included investigations of RA against different types of cancer [113-117]. Karmokar et al. showed that chronic consumption of RA $(0.3 \% \mathrm{w} / \mathrm{w}$ chow supplementation) for 8 weeks inhibited colon carcinogenesis in mice, and produced quantifiable levels of the parent compound in the plasma and the intestinal tract [118]. Additionally, RA suppressed retinal neovascularization [119], and exhibited transcorneal penetration when the RA-containing polyherbal formulation was instilled into rabbit eyes [120].

No increase was found in the frequency of micronuclei in mice treated orally with $50-200 \mathrm{mg} / \mathrm{kg}$ of RA, indicating that RA does not affect chromosomal integrity [121]. In fact, RA showed antimutagenic effects, protecting animals against doxorubicin [121], ethanol [122], and $\gamma$ ray-induced DNA damage [123], assessed by the comet and micronucleus assays using murine models.

Studies exploring the anti-inflammatory activity of RA are also frequent [124-127]. Youn et al. showed that RA (daily intraperitoneal injections, $50 \mathrm{mg} / \mathrm{kg}$ ) suppressed synovitis in a murine collagen induced arthritis model [128], while other groups reported the inhibition of allergic inflammatory reactions [129] and airway inflammation $[130,131]$ as well as atopic dermatitis [132, 133]. Jiang et al. reported that RA, administered intravenously (25 and $50 \mathrm{mg} / \mathrm{kg}$ ), either alone or in combination with imipenem, protected rats against experimental sepsis by decreasing local and systemic levels of an extensive spectrum of inflammatory mediators [134]. Additionally, oral administration of RA (2 mg/ body for 3 days) inhibited diesel exhaust particle-induced lung injury in mice by reducing the expression of proinflammatory molecules and antioxidative activities [135].

In regard to anti-infective potential, RA presented in vivo antiviral activity against the Japanese encephalitis virus, a member of the Flaviviruses family [136]. At 8 to 9 days postinfection, the mortality of mice treated intraperitoneally twice daily $(25 \mathrm{mg} /$ $\mathrm{kg}$ ) was reduced in comparison to untreated animals. This effect was mediated by decreased viral loads, and also by reduced levels of proinflammatory cytokines [136].

RA was also shown to be immunosuppressant, improving skin [137] and islet allograft survivals in murine models [138]. Antihemorrhagic, antithrombotic, and antiplatelet effects, along with the direct neutralization of multiple snake venoms and fish toxins, have also been reported for RA or RA-containing extracts [139-147], corroborating for a potentially beneficial role of RA for the treatment of poisoning accidents, already described in the in vitro section ( $\bullet$ Table 2 ).

Protective effects of RA in tissue or organs have been demonstrated. Domitrovic et al. showed that oral treatment with RA (1$5 \mathrm{mg} / \mathrm{kg}$ ) for two consecutive days improved cisplatin-induced kidney injury in mice by inhibiting oxidative stress, inflammation (TNF- $\alpha$ and NF- $k$ B), and apoptosis [148]. Other groups reported similar profiles [149-152], i.e., inhibition of diabetes-related renal damage [153] or inhibition of gentamicin sulphate-induced renal oxidative damage in rats treated orally with $50 \mathrm{mg} / \mathrm{kg}$ for 12 days $[154,155]$. Also, memory protective effects of RA in an Alzheimer's model ( $\beta$-amyloid neurotoxicity) was attributed to the radical scavenging potential of the molecule [156]. Cardioprotection [157,158], inhibition of endothelial tissue damage [159], and reduction of multiple organ failure in thermal injury [160] were linked to the antioxidant and anti-inflammatory effects of RA in vivo. Liver protection effects against cholestatic fibrosis, ischemia-reperfusion injury, $\mathrm{CCl}_{4}$, and lipopolysaccharide-induced damage were also reported at oral doses ranging from 2.5 to $50 \mathrm{mg} / \mathrm{kg}[160-165]$. 
Table 2 Summary of in vitro studies with isolated rosmarinic acid.

\begin{tabular}{|c|c|c|c|}
\hline First author, year (ref) & Method & Concentration & Main findings \\
\hline \multicolumn{4}{|l|}{ Anti-inflammatory } \\
\hline $\begin{array}{l}\text { Lembo et al., } 2014, \\
2011[225,226]\end{array}$ & $\begin{array}{l}\text { Cell viability (HaCaT cells) } \\
\text { Cytokine modulation by qRT-PCR }\end{array}$ & $2.7-55 \mu \mathrm{M}$ & prevention of UVB-induced inflammation \\
\hline Ku et al., 2013 [124] & ELISA (primary human umbilical vein endothelial cells) & $0.1-2 \mu \mathrm{M}$ & inhibition of endothelial protein C receptor shedding \\
\hline Moon et al., 2010 [227] & Cell viability (U937 human leukemia cells) & $15-60 \mu \mathrm{M}$ & $\begin{array}{l}\downarrow \text { TNF- } \alpha \text {-induced ROS generation } \\
\downarrow \text { NF- } \mathrm{B} \text { activation } \\
\uparrow \mathrm{TNF}-\alpha \text {-induced apoptosis }\end{array}$ \\
\hline Huang et al., 2009 [228] & LPS stimulation (RAW 264.7 mouse macrophage cells) & $2.67 \mu \mathrm{M}$ & $\begin{array}{l}\downarrow \text { LPS-induced prostaglandin E2 and nitric oxide } \\
\text { production }\end{array}$ \\
\hline $\begin{array}{l}\text { Zdarilová et al., } 2009 \\
\text { [229] }\end{array}$ & $\begin{array}{l}\text { LPS stimulation (primary cultured human gingival fi- } \\
\text { broblasts) }\end{array}$ & $1 \mu \mathrm{g} / \mathrm{mL}$ & $\begin{array}{l}\downarrow \text { oxidative damage } \\
\downarrow \text { inflammation }\end{array}$ \\
\hline Kim et al., 2008 [230] & $\begin{array}{l}\text { LPS inflammatory stimulation (BMDCs bone marrow- } \\
\text { derived dendritic cells) }\end{array}$ & $0.1-200 \mu \mathrm{M}$ & $\begin{array}{l}\downarrow \text { cell maturation and migration } \\
\downarrow \text { monocyte chemoattractant protein- } 1 \\
\downarrow \text { macrophage inflammatory protein- } 1 \alpha\end{array}$ \\
\hline $\begin{array}{l}\text { Scheckel et al., } 2008 \\
\text { [231] }\end{array}$ & $\begin{array}{l}\text { Luciferase assay (HT-29 colon cancer and MCF10 A } \\
\text { non-malignant epithelial cells) }\end{array}$ & $5-20 \mu \mathrm{M}$ & $\begin{array}{l}\downarrow \text { AP-1-dependent activation of cyclooxygenase- } 2 \\
\text { in human cancer and normal cells }\end{array}$ \\
\hline Lee et al., 2006 [232] & $\begin{array}{l}\text { ELISA and Western blotting (human dermal fibroblast } \\
\text { cells) }\end{array}$ & $\begin{array}{l}1-40 \mu \mathrm{M} \\
\mathrm{IC} \\
50=9.1-15.8 \mu \mathrm{M}\end{array}$ & $\begin{array}{l}\downarrow \text { expression of CCL11 and C-C motif chemokine } 11 \\
\text { receptor (CCR3) } \\
\downarrow \text { IKK- } \beta \text { activity }\end{array}$ \\
\hline \multicolumn{4}{|c|}{ Antiproliferative and Antimutagenic } \\
\hline Zhang et al., 2011 [233] & Cell viability (HSC-T6 hepatic stellate cells) & $\begin{array}{l}1-16 \mu \mathrm{g} / \mathrm{mL} \\
\mathrm{IC} \mathrm{C}_{50}=7.1 \mu \mathrm{g} / \mathrm{mL}\end{array}$ & $\begin{array}{l}\downarrow \text { cell proliferation } \\
\uparrow \text { apoptosis }\end{array}$ \\
\hline Xu et al., 2010 [113] & $\begin{array}{l}\text { Wound healing, adhesion and Transwell assays } \\
\text { (Ls174-T human colon carcinoma cells) }\end{array}$ & $\begin{array}{l}5-300 \mu \mathrm{g} / \mathrm{mL} \\
\mathrm{I} \mathrm{C}_{50}<20 \mu \mathrm{g} / \mathrm{mL}\end{array}$ & antimetastasis effect \\
\hline Xu et al., 2010 [234] & $\begin{array}{l}\text { Cell viability (MDA-MB-231BO human bone-homing } \\
\text { breast cancer cells) and wound healing assays }\end{array}$ & $1-300 \mu \mathrm{g} / \mathrm{mL}$ & $\begin{array}{l}\downarrow \text { tumoral cells migration }\left(\mathrm{IC}_{50}=118 \mu \mathrm{g} / \mathrm{mL}\right) \\
\text { bone protection in vitro }\end{array}$ \\
\hline Kim et al., 2009 [119] & $\begin{array}{l}\text { Cell viability and morphological changes (HRMEC hu- } \\
\text { man retina microvascular endothelial cells) }\end{array}$ & $10-100 \mu \mathrm{M}$ & $\begin{array}{l}\text { antiangiogenic activity } \\
\downarrow \text { cell proliferation } \\
\downarrow \text { tube formation }\end{array}$ \\
\hline $\begin{array}{l}\text { Xavier et al., 2008, } 2009 \\
{[235,236]}\end{array}$ & $\begin{array}{l}\text { Cell viability (HCT15 and CO115 human colorectal } \\
\text { carcinoma-derived cells) }\end{array}$ & $10-100 \mu \mathrm{M}$ & $\begin{array}{l}\downarrow \text { cell proliferation } \\
\uparrow \text { apoptosis }\end{array}$ \\
\hline $\begin{array}{l}\text { Hur et al., 2004, } 2007 \\
{[237,238]}\end{array}$ & $\begin{array}{l}\text { Flow cytometry (peripheral blood mononuclear cells } \\
\text { obtained from rheumatoid arthritis patients and Ju- } \\
\text { rkat acute T cell leukemia cells) }\end{array}$ & $3-100 \mu \mathrm{M}$ & $\begin{array}{l}\downarrow \text { cell proliferation } \\
\uparrow \text { apoptosis }\end{array}$ \\
\hline Huang et al., 2006 [239] & $\begin{array}{l}\text { Cell viability and migration assays (HUVEC human } \\
\text { umbilical vein endothelial cells) }\end{array}$ & $12.5-200 \mu \mathrm{M}$ & $\downarrow$ several steps of angiogenesis \\
\hline $\begin{array}{l}\text { Kolettas et al., } 2006 \\
{[240]}\end{array}$ & Cell viability (Jurkat human T lymphoma cells) & $10-150 \mu \mathrm{M}$ & $\begin{array}{l}\downarrow \text { cell proliferation } \\
\uparrow \text { apoptosis }\end{array}$ \\
\hline $\begin{array}{l}\text { Vattem et al., } 2006 \\
\text { [241] }\end{array}$ & AMES and supercoiled DNA strand scission assay & $500 \mu \mathrm{g} /$ plate & antimutagenic effect \\
\hline $\begin{array}{l}\text { Yoshida et al., } 2005 \\
\text { [242] }\end{array}$ & Cell viability (MK-1, HeLa, and B16F10 cells) & $\begin{array}{l}\text { MK-1, } \\
\mathrm{GI}_{50}=119 \mu \mathrm{M} \\
\text { HeLa, GI } \\
\text { B16 }=75 \mu \mathrm{M} 10 \\
\mathrm{GI}_{50}=16 \mu \mathrm{M}\end{array}$ & $\downarrow$ cell proliferation \\
\hline \multicolumn{4}{|l|}{ Cytoprotection } \\
\hline $\begin{array}{l}\text { Alcaraz et al., } 2014 \\
{[243]}\end{array}$ & $\begin{array}{l}\text { Micronucleus (human lymphocytes) } \\
\text { Cell viability (PNT2 and B16F10 cells) }\end{array}$ & $10-40 \mu \mathrm{M}$ & $\begin{array}{l}\text { radioprotection in normal cells } \\
\uparrow \text { radio-induced damage in melanoma cells }\end{array}$ \\
\hline Braidy et al., 2014 [244] & Cell viability (primary cultured human neurons) & $0.01-1 \mathrm{mg} / \mathrm{mL}$ & protection against ciguatoxin-induced neurotoxicity \\
\hline Costa et al., 2013 [245] & Cell viability (A172 human astrocyte cells) & $30 \mu \mathrm{g} / \mathrm{mL}$ & protection against $\mathrm{H}_{2} \mathrm{O}_{2}$-induced oxidative damage \\
\hline Jeon et al., 2013 [246] & Cell viability (primary cultured rat hepatocytes) & $1-100 \mu \mathrm{M}$ & cytoprotection against hypoxia-induced injury \\
\hline $\begin{array}{l}\text { Kim et al., } 2013 \text { [247]; } \\
\text { Jeong et al., } 2011 \text { [248] }\end{array}$ & $\begin{array}{l}\text { Cell viability (HEI-OC1 auditory cell line) } \\
\text { Fluorescence microscopy (Rat Corti primary explants) }\end{array}$ & $1-100 \mu \mathrm{M}$ & $\begin{array}{l}\text { protection against } \mathrm{Cd}^{+} \text {-induced or cisplatin-in- } \\
\text { duced ototoxicity } \\
\text { protection against cisplatin-induced destruction of } \\
\text { hair cell arrays ex vivo }\end{array}$ \\
\hline Yang et al., 2013 [249] & Cell viability (HepG2 human hepatoma cells) & $10-26.84 \mu \mathrm{g} / \mathrm{mL}$ & protection against $\mathrm{t}-\mathrm{BOOH}$-induced cytotoxicity \\
\hline $\begin{array}{l}\text { Du et al., } 2010 \text { [250]; } \\
\text { Ren et al., } 2009 \text { [251] }\end{array}$ & Cell viability (MES23.5 dopaminergic cells) & $0.001-100 \mu \mathrm{M}$ & $\begin{array}{l}\text { protection against 1-methyl-4-phenylpyridinium } \\
(\mathrm{MPP}+) \text { and 6-OHDA-induced neurotoxicity }\end{array}$ \\
\hline $\begin{array}{l}\text { Furtado et al., } 2010 \\
\text { [252] }\end{array}$ & Micronucleus and comet assay (V79 cells) & $0.28-1.12 \mathrm{mM}$ & $\begin{array}{l}\text { protection against doxorubicin-induced genotoxic- } \\
\text { ity }\end{array}$ \\
\hline $\begin{array}{l}\text { Fallarini et al., } 2009 \\
\text { [253] }\end{array}$ & $\begin{array}{l}\text { Cell viability [differentiated SH-SY5Y and SK-N-BE(2) } \\
\text { human neuroblastoma cells] }\end{array}$ & $\begin{array}{l}10-100 \mu \mathrm{M} \\
\mathrm{EC}_{50}=0.9-3.7 \mu \mathrm{M}\end{array}$ & $\begin{array}{l}\text { protection against t-BOOH-induced oxidative stress } \\
\downarrow \text { excitotoxicity } \\
\downarrow \text { ischaemia-reperfusion-induced neuronal death }\end{array}$ \\
\hline Lee et al., 2008 [254] & $\begin{array}{l}\text { Cell viability (SH-SY5Y human dopaminergic neuronal } \\
\text { cells) }\end{array}$ & $14-56 \mu \mathrm{M}$ & $\begin{array}{l}\text { protection against } \mathrm{H}_{2} \mathrm{O}_{2} \text {-induced neurotoxicity } \\
\downarrow \text { apoptosis }\end{array}$ \\
\hline
\end{tabular}


Table 2 Continued

\begin{tabular}{|c|c|c|c|}
\hline First author, year (ref) & Method & Concentration & Main findings \\
\hline $\begin{array}{l}\text { Salimei et al., } 2007 \\
\text { [255] }\end{array}$ & Cytofluorimetric approach (K562, NPA and ARO cells) & $25 \mu \mathrm{M}$ & $\downarrow$ sorbitol-induced apoptosis \\
\hline luvone et al., 2006 [256] & Cell viability (PC12 adrenal medulla cells) & $0.0036-36 \mu \mathrm{g} / \mathrm{mL}$ & $\downarrow \beta$-amyloid-induced cell death \\
\hline $\begin{array}{l}\text { Psotova et al., } 2006 \\
\text { [257] }\end{array}$ & Cell viability (HaCaT human keratinocyte cells) & $0.9-18 \mu \mathrm{g} / \mathrm{mL}$ & photoprotection against UVA-induced damage \\
\hline Yan et al., 2006 [258] & Cell viability (PC12 cells) & $100 \mu \mathrm{M}$ & $\begin{array}{l}\text { protection against glutamate-induced cell death } \\
\downarrow \text { apoptosis }\end{array}$ \\
\hline Gao et al., 2005 [259] & Cell viability (primary cultured rat astrocytes) & $10-40 \mu \mathrm{M}$ & $\begin{array}{l}\text { protection against } \mathrm{H}_{2} \mathrm{O}_{2} \text {-induced cell death } \\
\downarrow \text { apoptosis }\end{array}$ \\
\hline Kim et al., 2005 [260] & Cell viability ( $\mathrm{H} 9 \mathrm{c} 2$ cardiomyoblast cells) & $1-20 \mu \mathrm{g} / \mathrm{mL}$ & $\begin{array}{l}\text { protection against adriamycin-induced apoptosis } \\
\downarrow \text { apoptosis }\end{array}$ \\
\hline $\begin{array}{l}\text { Chlopcikova et al., } 2004 \\
\text { [261] }\end{array}$ & Cell viability (primary cultured rat cardiomyocytes) & $100-200 \mu \mathrm{M}$ & $\begin{array}{l}\text { protection against doxorubicin-induced cell death } \\
\downarrow \text { lipid peroxidation }\left(\mathrm{IC}_{50}=8.17 \mu \mathrm{M}\right)\end{array}$ \\
\hline $\begin{array}{l}\text { Renzulli et al., } 2004 \\
\text { [262] }\end{array}$ & Cell viability (Hep G2 human hepatoma-derived cells) & $2.5-100 \mu \mathrm{M}$ & $\begin{array}{l}\text { protection against aflatoxin B1 and ochratoxin A-in- } \\
\text { duced cytotoxicity }\end{array}$ \\
\hline \multicolumn{4}{|l|}{ Immunomodulation } \\
\hline Lee et al., 2007 [263] & $\begin{array}{l}\text { IFN- } \gamma \text { stimulation (BMDCs murine bone marrow-de- } \\
\text { rived dendritic cells) }\end{array}$ & $1-100 \mu \mathrm{M}$ & $\begin{array}{l}\downarrow \text { IDO-dependent T cell suppression } \\
\downarrow \text { functional expression of IDO }\end{array}$ \\
\hline $\begin{array}{l}\text { Ahn et al., } 2003 \text { [264]; } \\
\text { Kang et al., } 2003 \text { [265]; } \\
\text { Won et al., } 2003 \text { [266]; } \\
\text { Yun et al., } 2003 \text { [137] }\end{array}$ & $\begin{array}{l}\text { Binding assay } \\
\text { Cell proliferation and differentiation }\end{array}$ & $\begin{array}{l}0.1-1000 \mu \mathrm{M} \\
\mathrm{IC}_{50}=5.6-14 \mu \mathrm{M}\end{array}$ & $\begin{array}{l}\text { inhibition of lymphocyte cell-specific kinase Src-ho- } \\
\text { mology } 2 \text { domain binding } \\
\downarrow T \text { cell activation } \\
\downarrow T \text { cell proliferation }\end{array}$ \\
\hline Kang et al., 2003 [267] & Luciferase and $\mathrm{Ca}^{2+}$ mobilization assays (Jurkat T cells) & $5-30 \mu \mathrm{M}$ & $\begin{array}{l}\downarrow \mathrm{Ca}^{2+} \text {-dependent pathways of T-cell antigen re- } \\
\text { ceptor-mediated signalling }\end{array}$ \\
\hline $\begin{array}{l}\text { Sahu et al., } 1999 \text { [268]; } \\
\text { Peake et al., } 1991 \text { [269] }\end{array}$ & $\begin{array}{l}\text { Binding assays } \\
\mathrm{C} 3 \text { and } \mathrm{C} 5 \text { convertase assays }\end{array}$ & $0.01-10 \mathrm{mM}$ & $\begin{array}{l}\downarrow \text { complement activation } \\
\text { binding to activated C } 3 \mathrm{~b} \\
\text { C5 convertase inhibition }\end{array}$ \\
\hline \multicolumn{4}{|l|}{ Miscelaneous } \\
\hline $\begin{array}{l}\text { Abedini et al., } 2013 \\
\text { [270] }\end{array}$ & $\begin{array}{l}\text { Minimal inhibitory concentration/minimal bacterici- } \\
\text { dal concentration by broth microdilution }\end{array}$ & $\begin{array}{l}0.0093-2.5 \mathrm{mg} / \\
\mathrm{mL}\end{array}$ & antimicrobial activity \\
\hline Airoldi et al., 2013 [271] & NMR spectroscopy & $1 \mathrm{mM}$ & $\begin{array}{l}\text { binding to } \beta \text {-amyloid oligomers (Alzheimer's dis- } \\
\text { ease) }\end{array}$ \\
\hline $\begin{array}{l}\text { Marcelo et al., } 2013 \\
\text { [272]; Yin et al., } 2008 \\
\text { [273] }\end{array}$ & $\begin{array}{l}\text { NMR spectroscopy } \\
\text { Molecular Modelling }\end{array}$ & $\begin{array}{l}333 \mu \mathrm{M} \\
50 \mathrm{mM}\end{array}$ & $\begin{array}{l}\text { binding to Acetylcholinesterase (Alzheimer's dis- } \\
\text { ease) }\end{array}$ \\
\hline $\begin{array}{l}\text { Slobodníková et al., } \\
2013 \text { [274] }\end{array}$ & $\begin{array}{l}\text { Minimal inhibitory concentration/minimal bacterici- } \\
\text { dal concentration by broth microdilution } \\
\text { Biofilm regrowth technique }\end{array}$ & $156-5000 \mu \mathrm{g} / \mathrm{mL}$ & $\begin{array}{l}\text { antimicrobial activity } \\
\text { inactive as biofilm-eradicator }\end{array}$ \\
\hline Yang et al., 2012 [161] & $\begin{array}{l}\text { Cellular differentiation reversal } \\
\text { (primary cultured hepatic stellate cells) }\end{array}$ & $135-270 \mu \mathrm{M}$ & $\begin{array}{l}\text { antifibrotic effect } \\
\downarrow \text { epigenetic peroxisomal proliferator-activated re- } \\
\text { ceptor } \gamma \text { repression } \\
\text { reversal of activated cellular phenotypes }\end{array}$ \\
\hline $\begin{array}{l}\text { Dos Santos et al., 2010, } \\
2011 \text { [142, 143]; Ticli et } \\
\text { al., } 2005 \text { [144] }\end{array}$ & $\begin{array}{l}\text { Phospholipase }{ }_{2} \text { enzymatic activity inhibition } \\
\text { Molecular modelling } \\
\text { Muscle-damaging and neuromuscular-blocking activ- } \\
\text { ities }\end{array}$ & $13.7 \mu \mathrm{g} / \mathrm{mL}$ & antivenom effects in vitro and ex vivo \\
\hline Lin et al., 2011 [275] & Enzymatic activity inhibition & $0.01-0.4 \mathrm{mM}$ & tyrosinase and $\alpha$-glucosidase inhibition \\
\hline $\begin{array}{l}\text { Murata et al., } 2011 \\
\text { [276]; Ippoushi et al., } \\
2000 \text { [277] }\end{array}$ & Enzymatic activity inhibition & $\begin{array}{l}0.03-1 \mathrm{mM} \\
\mathrm{IC}_{50}=309 \mu \mathrm{M}\end{array}$ & hyaluronidase inhibition \\
\hline $\begin{array}{l}\text { Falé et al., 2008, } 2009 \\
{[278,279]}\end{array}$ & Enzymatic activity inhibition & $\mathrm{IC}_{50}=440 \mu \mathrm{g} / \mathrm{mL}$ & acetylcholinesterase inhibition (Alzheimer's disease) \\
\hline Lee et al., 2007 [280] & Melanin content determination (B16 melanoma cells) & $1-100 \mu \mathrm{M}$ & $\uparrow$ melanogenesis \\
\hline Kang et al., 2004 [281] & Enzymatic activity inhibition & $\mathrm{IC}_{50}=16.8 \mu \mathrm{M}$ & tyrosinase inhibition \\
\hline $\begin{array}{l}\text { McCue et al., } 2004 \\
\text { [282] }\end{array}$ & Enzymatic activity inhibition & $0.07-0.42 \mathrm{mM}$ & amylase inhibition \\
\hline $\begin{array}{l}\text { Makino et al., } 2000 \\
\text { [151] }\end{array}$ & Cell viability (primary cultured murine mesangial cells) & $\begin{array}{l}1-25 \mu \mathrm{g} / \mathrm{mL} \\
\mathrm{IC}_{50}=1.4-3.8 \mu \mathrm{g} / \\
\mathrm{mL}\end{array}$ & $\downarrow$ cell proliferation \\
\hline $\begin{array}{l}\text { Simpol et al., } 1994 \\
{[283]}\end{array}$ & Histamine release inhibition (rat mast cells) & $\mathrm{IC}_{50}=18 \mu \mathrm{M}$ & $\downarrow$ histamine release \\
\hline
\end{tabular}

$\uparrow$ Increase; $\downarrow$ decrease/inhibition; NA - not available; Most studies used multiple assays/endpoints for the pharmacological evaluation of RA; For a complete methodological assessment, please refer to the original article 
The neuroprotective effect of RA was studied in animal models of central nervous system diseases. Mushtaq et al. showed that oral treatment with RA ( $10 \mathrm{mg} / \mathrm{kg}$ ) for 21 days significantly reduced the level of lipid peroxidation in multiple areas of the brain in diabetic rats, together with modulation of cholinergic neurotransmission [166]. At doses higher than $50 \mathrm{mg} / \mathrm{kg}$, RA produced a significant anti-inflammatory effect in rats submitted to an experimental ischemic diabetic stroke model [167]. In a Parkinson's disease model in mice, 6-OHDA-induced degeneration of the nigrostriatal dopaminergic system was reversed by 21 days of oral treatment with RA at $20 \mathrm{mg} / \mathrm{kg}$ [168]. A number of groups reported that administration of RA (orally or intraperitoneally) alleviated stress symptoms [169-173] and depressive-like behaviors [174-176], and produced anxiolytic-like effects, without exerting locomotor alterations or DNA damage in brain tissue [177]. Additionally, subchronic oral RA treatment of mice (up to 3 weeks) has a cognitive-enhancing effect [178], and, in a model of Amyotrophic lateral sclerosis, RA administered intraperitoneally $(0.13 \mathrm{mg} / \mathrm{kg}$, twice a week) significantly delayed motor dysfunction, attenuated motor neuron degeneration, and improved clinical outcomes and lifespan [179].

Finally, an unusual preclinical study in a Rhesus monkey model was conducted to determine the effect of the topical application of RA on the progression of plaque-induced gingivitis. Six young adult (4-6 years) male Rhesus monkeys (Macaca mulatta) randomly assigned to 3 groups of 2 each (A, B and C) received a topical application of vehicle alone, the drug ebselen (1\%), and RA (5\%) twice weekly for two weeks. To promote plaque retention, the chow was softened with water prior to feeding. Clinical evidence of gingivitis (erythema, edema) was apparent after 2-3 days, with established gingivitis $(\mathrm{GI}=2)$ apparent in all animals after one week. Based on these preliminary studies, the authors concluded that at least in the short term, ebselen and RA are effective at reducing both gingival inflammation and plaque accumulation when topically applied [180].

\section{Clinical studies with rosmarinic acid containing extracts}

As a widespread metabolite, several traditional plants have been clinically studied using RA as a chemical marker or as an active compound. Different clinical protocols designed to assess the anti-inflammatory effects and the antioxidant potential of plants containing RA have been employed.

Initially, links between oxidative stress and adverse health effects have been suggested; for example, Ranjbar et al. [181] investigated the antioxidant influence of an Echium amoenum Fisch \& C. A. Mey (Boraginaceae) decoction in healthy volunteers using a cross-sectional before/after clinical trial. The authors considered E. amoenum, one of the most important medicinal plants in Iranian traditional medicine (despite its hepatotoxic pyrrolizidine alkaloids [182]) to be a rich source of RA and flavonoids. They randomly selected thirty-eight volunteers (18-25 years old), students at Arak University of Medical Sciences, who were interviewed by a specialized physician before receiving the E. amoenum flower decoction $(7 \mathrm{mg} / \mathrm{kg}$ ) twice daily (morning and evening) for 2 weeks. The authors found a significant decrease $(\mathrm{p}<0.05)$ in lipid peroxidation $(24.65 \pm 11.33$ to $19.05 \pm$ $9.7 \mathrm{nmol} / \mathrm{mL})$, an increase in total blood antioxidant capacity $(\mathrm{p}<0.05 ; 1.46 \pm 0.51$ to $1.70 \pm 0.36 \mathrm{mmol} / \mathrm{mL})$, as well as an increase $(p<0.001)$ in total thiol molecules $(0.49 \pm 0.11$ to $0.56 \pm 0.12 \mathrm{mmol})$, after administration of the decoction. In their view, this is the mechanism by which RA and antioxidants protect the human body from various diseases. This study has a ma- jor limitation, as the authors administered a decoction that was not standardized in terms of RA content.

With a similar objective, a crossover randomized controlled trial study [183] detected, by means of LC-quadrupole time-of-flight, phenolic acids (among them RA) and aromatic compounds in human plasma $24 \mathrm{~h}$ after consumption of a blueberry drink containing $766 \mathrm{mg}$ total polyphenols by healthy volunteers $(n=10)$. The primary outcome was the measure of flow-mediated dilation. Flow-mediated dilation increased after $1 \mathrm{~h}$ of consumption and then plateaued. According to the authors, increases in flow-mediated dilation were closely linked to increases in circulating phenolic metabolites and decreases in neutrophil NADPH oxidase activity at 1-2 and $6 \mathrm{~h}$. The authors reported limitations when interpreting the trial datasets, such as the short time frame, causality, and the population studied.

Also focusing on antioxidant foods, a study was conducted with 11 healthy volunteers consuming two kinds of burger meat seasoned or unseasoned with a spice blend [184]. The production of malondialdehyde in the burgers and the malondialdehyde concentration in plasma and urine after ingestion were measured, since the formation of malondialdehyde has implications for atherogenesis and carcinogenesis [185]. RA from oregano was monitored to assess the effect of cooking on the spice antioxidant content. Forty percent (19 mg) of the rosmarinic acid added remained in the spiced burger after cooking. There was a $71 \%$ reduction in the malondialdehyde concentration $(0.52 \pm 0.02 \mu \mathrm{mol} /$ $250 \mathrm{~g}$ ) in the meat of the spiced burgers compared with the malondialdehyde concentration $(1.79 \pm 0.17 \mu \mathrm{mol} / 250 \mathrm{~g})$ in the meat of the control burgers. The plasma malondialdehyde concentration increased significantly in the control burger group compared to the baseline $(p=0.026)$. There was a significant time-trend difference ( $p=0.013$ ) between the two groups. Urinary malondialdehyde concentrations ( $\mu \mathrm{mol} / \mathrm{g}$ creatinine) decreased by $49 \%$ $(p=0.021)$ in subjects consuming the spiced burgers compared with subjects consuming the control burgers. The authors concluded that cooking hamburgers with a polyphenol-rich spice mixture can significantly decrease the concentration of malondialdehyde, suggesting potential health benefits for atherogenesis and carcinogenesis.

Nasal polyposis is a mucosal inflammatory disease that was investigated in a double-blind placebo-controlled crossover trial using 1 cup of a mint tea high in RA (about $300 \mathrm{mg}$ ) versus 1 cup of a mint tea low in RA (about $20 \mathrm{mg}$ per day). Each treatment period lasted 4 weeks, separated by a 4 -week washout period, and the first treatment followed a 2-week baseline period. Twentytwo adult subjects completed the study, and the authors found no statistically significant difference between the treatments in nasal stuffiness, as recorded on daily diary cards, in peak nasal inspiratory flows measured twice daily, or in patients' global assessment (including ability to smell or sleep) performed at the end of each treatment period [186].

A more recent randomized, parallel-arm, double-blind study (meeting the CONSORT statement) investigated the effect of RA in the management of knee osteoarthritis symptoms using tea brewed from the high-RA spearmint plant (130-150 mg of RA per cup, $\mathrm{n}=22$ ) and a commercially available spearmint tea (ca $13 \mathrm{mg}$ of RA, $\mathrm{n}=24$ ). The subjects were instructed to consume two cups of tea per day from a $300-\mathrm{mL}$ study mug provided to them, for a 16 -week period. The outcome was measured using the Western Ontario and McMaster Universities Osteoarthritis (WOMAC) pain score, which is a validated, standardized 24-item questionnaire that assesses pain, disability, and joint stiffness as- 
sociated with osteoarthritis. The study allowed the subjects to maintain their normal pain medication in order to explore the potential of the high-RA tea as a complementary therapy. The authors concluded that individuals who consumed $600 \mathrm{~mL}$ of highRA spearmint tea daily showed a significant decrease in pain scores from weeks 0 to 16 , inferring that adults with knee osteoarthritis may benefit from the inclusion of high-RA spearmint tea in their daily diet. No serious adverse events were reported during the study [187].

\section{Clinical studies with isolated rosmarinic acid}

RA was clinically investigated in atopical dermatitis due to its in vitro and in vivo anti-inflammatory effects, such as its ability to block complement fixation, inhibit lipoxygenase and cyclooxygenase activity, and suppress IKK- $\beta$ downstream signaling in the TNF- $\alpha$-induced upregulation of CCL11. Oil-in-water cream with or without rosmarinic acid $(0.3 \%)$ was applied to the elbow flexures of 21 subjects ( 14 women and seven men; $15.1 \pm 3.1$ years) twice daily, for 4 and 8 weeks. The subjects were clinically graded as having moderate atopic dermatitis, according to the guidelines of the SCORAD index. The evaluation methods included clinical assessments (SCORAD), instrumental assessments (TEWL) and self-assessments by questionnaire. The authors reported that RA cream caused no reactions in the patch test on patient, suggesting that RA can safely be applied to human skin. After treatment with RA, the authors observed a statistically significant reduction in the SCORAD score, a decrease in itching, and a decrease in TEWL, suggesting that RA is a possible atopical dermatitis-mitigating agent [188].

Likewise, considering that RA is a strong anti-inflammatory agent in several animal models, a 21-day randomized controlled trial was undertaken to determine whether oral RA supplementation [ $200 \mathrm{mg}(\mathrm{n}=10)$ or $50 \mathrm{mg}(\mathrm{n}=9)$ ] is an effective intervention for patients with seasonal allergic rhinoconjuctivitis. The patients recorded their symptoms daily in a diary card, and profiles of infiltrating cells, concentrations of eotaxin, IL- $1 \beta$, IL-8, and histamine in nasal lavage fluid, as well as serum IgE concentrations, were measured. The authors found that RA supplementation resulted in a significant increase in responder rates for itchy nose, watery eyes, itchy eyes, and overall symptoms ( $\mathrm{p}<0.05)$. Additionally, the treatment significantly decreased the numbers of neutrophils and eosinophils in nasal lavage fluid, compared to placebo supplementation. Patients reported no adverse events, and no significant abnormalities were detected in routine blood tests [189].

\section{Pharmacokinetics in Humans}

$\nabla$

Pharmacokinetic studies designed for healthy volunteers have been described. A randomized, open-label, single-dose study investigated the PK parameters of RA and other depside salts after intravenous infusion in 12 volunteers ( 6 male, 6 female) divided into two groups, randomly receiving either 100 or $200 \mathrm{mg}$ of RA. The authors described no significant differences in the PK parameters between male and female subjects. Three undetermined metabolites were found in the plasma at low concentrations. The urinary excretion recoveries of RA were $25.21 \%$ (20.61\%) for the $100 \mathrm{mg}$ dose, and $20.11 \%$ (10.50\%) for the $200 \mathrm{mg}$ dose. No adverse events were reported by the subjects or found by the investigators [190]. In a crossover design, a PK study with six healthy male volunteers with a 10-day washout period between the administration of $P$. frutescens extract in a tablet containing $20 \%$ RA and placebo was carried out. The authors found that RA reached a maximum concentration in plasma after $0.5 \mathrm{~h}$, followed by a gradual increase in the plasma concentration of methyl-RA, which reached a peak by $2 \mathrm{~h}$. Approximately $75 \%$ of the total RA metabolites were then excreted in the urine within $6 \mathrm{~h}$. These results showed that RA contained in P. frutescens extract was rapidly absorbed, subsequently methylated, and then excreted in the urine, and the majority of RA and its metabolites were present in the plasma as conjugated forms. The main metabolites found in the urine after consuming $P$. frutescens extract were sulfoglucuronide conjugates of RA and methyl-RA, indicating that conjugation of polyphenolic substances, either by glucuronidation and/or sulfation, occurs in human tissues such as the intestine and liver [191]. In comparative studies, Nakazawa and Ohsawa [192,193] found differences between human and rat metabolites of RA, reporting that RA may be predominantly metabolized to trimethoxycinnamic acid monoglucuronide in humans through hydrolytic cleavage in the gut, whereas in rats, RA is mainly metabolized to sulfated forms of trans-caffeic, ferulic, and m-coumaric acids.

\section{Pharmacokinetics in Animals $\nabla$}

Pharmacokinetic studies have been conducted in rats. Azevedo et al. showed that RA can modulate the transit of the intestinal $\mathrm{Na}+$ /glucose cotransporter-1 (SGLT-1) to the brush-border membrane, an effect that may contribute to the control of plasma glucose levels in diabetic rats [194]. Additionally, Debersac et al. showed that supplementation of a rat diet with $0.5 \%$ RA for 2 weeks did not increase the liver cytochrome P450 enzyme levels [195].

Konishi et al. compared PK parameters of caffeic acid and RA after oral administration in rats, indicating that the absorption efficiency of caffeic acid was higher than that of RA, and that the conjugation of both compounds occurs during permeation across the rat epithelium [196]. These findings are in accordance with Baba et al., which demonstrated that RA was rapidly absorbed (plasma values ca. $5 \mu \mathrm{mol} / \mathrm{L}$ ) and metabolized into conjugated and/or methylated forms, and a large amount of the absorbed RA was degraded and metabolized as conjugated forms of caffeic, ferulic, and m-coumaric acids [197].

Two studies on the development of the LC-MS/MS method and its application to PK studies of RA in rats found similar values for RA PK parameters, such as $C_{\max }(48.67 \pm 11.24$ [198] and 37.19 \pm $13.85 \mathrm{ng} / \mathrm{mL}$ [199]) and $\mathrm{T}_{\max }(1.08 \pm 0.38$ and $0.74 \pm 0.12 \mathrm{~h}$ [199]), despite using different mathematical methods and doses. In [198], Drug and Statistics (DAS) 2.0 software (non-compartmental), and in [199], the practical pharmacokinetic program version 87 (3P87) (survival square sum) were used, with a single administration of Herba Isodi Rubescentis extract containing RA $2.55 \mathrm{mg} / \mathrm{kg}$ in [198], and in [199], a mixture of the standards in saline, with RA at $6.39 \mathrm{mg} / \mathrm{kg}$.

\section{Pharmaceutical Development \\ $\nabla$}

Focusing on the potential antioxidant activity of RA, technological studies were conducted with the aim of improving stability and bioavailability, and verifying antioxidant interactions. The incorporation of RA into formulas as an active component was al- 
so proposed. In order to overcome the limitations of RA for cosmetic purposes, such as its low water solubility and discoloration, polycaprolactone microspheres loaded with RA were developed. RA-polycaprolactone microspheres with zwitterionic and nonionic surfactants presented better loading efficiency when $10 \mathrm{mg}$ of RA was incorporated. The microspheres showed better long-term stability in a cream formulation compared to RA alone [200]. In another study, solid lipid nanoparticles loaded with RA were prepared with the same objectives, resulting in better stability, which can be useful for applications in the food industry [201]. Also with the aim of increasing stability and water solubility, the cyclodextrin $(\beta-C D)$ complexation of RA improved the properties and antioxidant capacity [202,203]. To study the interactions with $\alpha$-tocopherol, the antioxidant efficiency of RA and RA-esters in oil-water emulsions was measured. All combinations resulted in improved antioxidant activity when compared to isolated compounds, since the concentrations were higher, and the combination of $\alpha$-tocopherol and RA presented a synergistic effect. According to the authors, the formation of caffeic acid from RA in the presence of $\alpha$-tocopherol occurred, and this conversion provided additional antioxidant outcomes [204]. RA proposed as an active compound against Acne vulgaris has been incorporated in a niosomal gel tested in vivo (Swiss albino mice). Both plain and niosomal formulations inhibited inflammation, and only the niosomal gel reduced the bacterial multiplication rate 4 days after application, due to prolonged release [205]. Also, considering the anti-inflammatory potential of RA, a preparation of a polygalacturonic/RA biodegradable membrane was developed to prevent postoperative abdominal adhesion, and was evaluated in vitro and in vivo. The authors demonstrated that this membrane could effectively inhibit adhesion as well as acute and chronic inflammation in rats [206].

Focusing on the construction of biosensors with high analytical performance, RA was used as a model for the quantification of pharmaceutical samples in an optimized biosensor synthetized with gold nanoparticles in an ionic liquid phase, supported in a biopolymeric matrix. The developed biosensor offered good precision and accuracy for the determination of RA [207]. In another study, RA was used to generate and stabilize gold and silver nanoparticules, acting as an appropriate antioxidant, being able to reduce the metallic ions, and also acting as a surface-passivation agent [208].

\section{Concluding Remarks \\ $\nabla$}

RA is a natural metabolite that has attracted the interest of researchers in various areas of knowledge due to its abundance and potential biological properties. Analytical, biological, and technological RA data are compiled here for the first time.

It has served as a model for a series of scientific proposals, and will continue to be a target of investigations and the production of knowledge. There is still much that needs to be done to ensure the data from nonclinical to clinical trials are translated into meaningful knowledge. There is a lack of connectivity between studies, and many gaps are yet to be filled in order to authenticate the role of RA in human health. In future investigations covering RA, translational research should be accomplished: the application of the findings generated during laboratory or preclinical research to the development of trials and studies in humans, furnishing promising new treatments with practical applications.

\section{Methodology}

$\nabla$

Relevant articles from the literature were collected by searching the main scientific databases including Pubmed, Scopus, Scifinder, Web of Science, and Science Direct from 1990-2015, limiting the search to the occurrence of the keywords "Rosmarinic acid" in the title. This preliminary search identified more than 800 articles, which after the initial screening and removal of duplicates was refined, resulting in 281 articles that were scrutinized to compose this review.

\section{Acknowledgements}

$\nabla$

The authors are grateful to CNPq for the grants and scholarships.

\section{Conflict of Interest \\ $\nabla$}

The authors declare no conflicts of interest.

\section{References}

1 Khojasteh A, Mirjalili MH, Hidalgo D, Corchete P, Palazon J. New trends in biotechnological production of rosmarinic acid. Biotechnol Lett 2014; 36: 2393-2406

2 Petersen M. Rosmarinic acid: new aspects. Phytochem Rev 2013; 12 : 207-227

3 Bulgakov VP, Inyushkina YV, Fedoreyev SA. Rosmarinic acid and its derivatives: biotechnology and applications. Crit Rev Biotechnol 2012; 32: 203-217

4 Petersen M, Abdullah Y, Benner J, Eberle D, Gehlen K, Hücherig S, Janiak V, Kim KH, Sander M, Weitzel C. Evolution of rosmarinic acid biosynthesis. Phytochemistry 2009; 70: 1663-1679

5 Park SU, Uddin R, Xu H, Kim YK, Lee SY. Biotechnological applications for rosmarinic acid production in plant. Afr J Biotechnol 2008; 7: 49594965

6 Petersen M, Simmonds MS. Rosmarinic acid. Phytochemistry 2003; 62: $121-125$

7 Akowuah GA, Ismail Z, Norhayati I, Sadikun A. The effects of different extraction solvents of varying polarities on polyphenols of Orthosiphon stamineus and evaluation of the free radical-scavenging activity. Food Chem 2005; 93: 311-317

8 Zu G, Zhang R, Yang L, Ma C, Zu Y, Wang W, Zhao C. Ultrasound-assisted extraction of carnosic acid and rosmarinic acid using ionic liquid solution from Rosmarinus officinalis. Int J Mol Sci 2012; 13: 11027-11043

9 Dent M, Dragovic-Uzelac V, Penic M, Brncic M, Bosiljkov T, Levaj B. The effect of extraction solvents, temperature and time on the composition and mass fraction of polyphenols in Dalmatian wild sage (Salvia officinalis L.) extracts. Food Technol Biotechnol 2013; 51: 84-91

10 Argoti JC, Linares-Palomino PJ, Salido S, Ramirez B, Insuasty B, Altarejos J. On-line activity screening for radical scavengers from Baccharis chilco. Chem Biodivers 2013; 10: 189-197

11 Satake T, Kamiya K, Saiki Y, Hama T, Fujimoto U, Kitanaka S, Kimura Y, Uzawa J, Endang H, Umar M. Studies on the constituents of fruits of $\mathrm{Hel}$ icteres isora L. Chem Pharm Bull 1999; 47: 1444-1447

12 de Oliveira $K B$, de Oliveira BH. HPLC/DAD determination of rosmarinic acid in Salvia officinalis: sample preparation optimization by factorial design. J Braz Chem Soc 2013; 24: 85-91

13 Wang L, Wang S, Yang S, Guo X, Lou H, Ren D. Phenolic alkaloids from the aerial parts of Dracocephalum heterophyllum. Phytochemistry 2012; 82: $166-171$

14 Takeda Y, Ooiso Y, Masuda T, Honda G, Otsuka H, Sezik E, Yesilada E. Iridoid and eugenol glycosides from Nepeta cadmea. Phytochemistry 1998; 49: 787-791

15 Wang MF, Li JG, Rangarajan M, Shao Y, LaVoie EJ, Huang TC, Ho CT. Antioxidative phenolic compounds from sage (Salvia officinalis). J Agric Food Chem 1998; 46: 4869-4873

16 Adham AN. Comparative extraction methods, phytochemical constituents, fluorescence analysis and HPLC validation of rosmarinic acid con- 
tent in Mentha piperita, Mentha longifolia and Osimum basilicum. J Pharmacogn Phytochem 2015; 3: 130-139

17 Abdullah Y, Schneider B, Petersen M. Occurrence of rosmarinic acid, chlorogenic acid and rutin in Marantaceae species. Phytochem Lett 2008; 1: 199-203

18 Aritomi M, Kumori T, Kawasaki T. Cyanogenic glycosides in leaves of Perilla-Frutescens var. acuta (Chemical studies on the constituents of edible plants, Part 4). Phytochemistry 1985; 24: 2438-2439

19 Ondrejovič $M$, Benkovičová $H$, Šilhár S. Optimization of rosmarinic acid extraction from lemon balm (Melissa officinalis). Nova Biotechnol 2009; 9-2: 175-182

20 Zibetti A, Aydi A, Livia M, Bolzan A, Barth D. Solvent extraction and purification of rosmarinic acid from supercritical fluid extraction fractionation waste: Economic evaluation and scale-up. J Supercrit Fluids 2013; 83: 133-145

21 Angelov G, Penchev P, Condoret JS. Extraction of rosmarinic acid from botanicals with supercritical carbon dioxide. Effect of the modifiers added to the solvent. C R Acad Bulg Sci 2011; 64: 953-958

22 Ho SK, Tan CP, Thoo YY, Abas F, Ho CW. Ultrasound-assisted extraction of antioxidants in Misai Kucing (Orthosiphon stamineus). Molecules 2014; 19: 12640-12659

23 Dragović-Uzelac V, Garofulić IE, Jukić M, Penić M, Dent $M$. The influence of microwave-assisted extraction on the isolation of sage (Salvia officinalis L.) Polyphenols. Food Technol Biotechnol 2012; 50: 377-383

24 Hong E, Park KH, Kim GH. Phenolic-enriched fractions from Perilla frutescens var. acuta: Determinating rosmarinic acid and antioxidant activity. J Food Biochem 2011; 35: 1637-1645

25 Cserháti T. Carbon-based sorbents in chromatography. New achievements. Biomed Chromatogr 2009; 23: 111-118

26 Kouri G, Tsimogiannis D, Bardouki H, Oreopouiou V. Extraction and analysis of antioxidant components from Origanum dictamnus. Innov Food Sci Emerg Technol 2007; 8: 155-162

27 Kim S, Yun EJ, BakJS, Lee H, Lee SJ, Kim CT, Lee JH, Kim KH. Response surface optimised extraction and chromatographic purification of rosmarinic acid from Melissa officinalis leaves. Food Chem 2010; 121: 521-526

28 Hossain MA, Salehuddin SM, Kabir MJ, Rahman SMM, Rupasinghe HPV. Sinensetin, rutin, 3'-hydroxy-5,6,7,4'-tetramethoxyflavone and rosmarinic acid contents and antioxidative effect of the skin of apple fruit. Food Chem 2009; 113: 185-190

29 Sahin S, Aybastier O, Isik E. Optimisation of ultrasonic-assisted extraction of antioxidant compounds from Artemisia absinthium using response surface methodology. Food Chem 2013; 141: 1361-1368

30 Hossain MB, Brunton NP, Martin-Diana AB, Barry-Ryan C. Application of response surface methodology to optimize pressurized liquid extraction of antioxidant compounds from sage (Salvia officinalis L.), basil (Ocimum basilicum L.) and thyme (Thymus vulgaris L.). Food Funct 2010; 1: 269-277

31 Hossain MB, Brunton NP, Patras A, Tiwari B, O'Donnell CP, Martin-Diana $A B$, Barry-Ryan C. Optimization of ultrasound assisted extraction of antioxidant compounds from marjoram (Origanum majorana L.) using response surface methodology. Ultrason Sonochem 2012; 19: 582-590

32 Herrero M, Arraez-Roman D, Segura A, Kenndler E, Gius B, Raggid MA, Ibanez E, Cifuentes A. Pressurized liquid extraction-capillary electrophoresis-mass spectrometry for the analysis of polar antioxidants in rosemary extracts. J Chromatogr A 2005; 1084: 54-62

33 Liu F, Tian S. A rapid method for the determination of rosmarinic acid in Cordia dichotoma seeds using ultrasound-assisted extraction and highperformance liquid chromatography-tandem mass spectrometry. Res J Chem Environ 2013; 17: 145-152

34 Paniwnyk L, Cai H, Albu S, Mason TJ, Cole R. The enhancement and scale up of the extraction of anti-oxidants from Rosmarinus officinalis using ultrasound. Ultrason Sonochem 2009; 16: 287-292

35 Vicente G, Molina S, González-Vallinas M, García-Risco MR, Fornari T, Reglero G, De Molina AR. Supercritical rosemary extracts, their antioxidant activity and effect on hepatic tumor progression. J Supercrit Fluids 2013; 79: 101-108

36 Lung I, Soran ML, Tudoran C, Marutoiu C. Effect of microwave irradiation on polyphenolic compounds from Satureja hortensis L. Cent Eur J Chem 2013; 11: 535-541

37 Rodríguez-Rojo S, Visentin A, Maestri D, Cocero MJ. Assisted extraction of rosemary antioxidants with green solvents. J Food Eng 2012; 109: 98-103

38 Liu T, Sui X, Zhang R, Yang L, Zu Y, Zhang L, Zhang Y, Zhang Z. Application of ionic liquids based microwave-assisted simultaneous extraction of carnosic acid, rosmarinic acid and essential oil from Rosmarinus officinalis. J Chromatogr A 2011; 1218: 8480-8489

39 Ozyurek M, Bener M, Guclu K, Apak R. Antioxidant/antiradical properties of microwave-assisted extracts of three wild edible mushrooms. Food Chem 2014; 157: 323-331

40 Orlowska M, Stanimirova I, Staszek D, Sajewicz M, Kowalska T, Waksmundzka-Hajnos M. Optimization of extraction based on the thin-layer chromatographic fingerprints of common thyme. J AOAC Int 2014; 97 : 1274-1281

41 Miron TL, Herrero M, Ibanez E. Enrichment of antioxidant compounds from lemon balm (Melissa officinalis) by pressurized liquid extraction and enzyme-assisted extraction. J Chromatogr A 2013; 1288: 1-9

42 Chatterjee A, Tandon S, Ahmad A. Comparative extraction and downstream processing techniques for quantitative analysis of rosmarinic acid in Rosmarinus officinalis. Asian J Chem 2014; 26: 4313-4318

43 Peshev D, Peeva LG, Peev G, Baptista IIR, Boam AT. Application of organic solvent nanofiltration for concentration of antioxidant extracts of rosemary (Rosmarinus officinalis L.). Chem Eng Res Des 2011; 89: 318-327

44 Peev G, Penchev P, Peshev D, Angelov G. Solvent extraction of rosmarinic acid from lemon balm and concentration of extracts by nanofiltration: Effect of plant pre-treatment by supercritical carbon dioxide. Chem Eng Res Des 2011; 89: 2236-2243

45 Rahimi A, Hashemi P, Badiei A, Safdarian M, Rashidipour M. Microextraction of rosmarinic acid using CMK-3 nanoporous carbon in a packed syringe. Chromatographia 2013; 76: 857-860

$46 \mathrm{Hu}$ SS, Cao W, Da JH, Dai HB, Cao J, Ye LH, Li XY, Chu C. Dispersive micro solid-phase extraction with graphene oxide for the determination of phenolic compounds in dietary supplements by ultra high performance liquid chromatography coupled with quadrupole time-of-flight tandem mass spectrometry. Food Anal Methods 2015; 8: 833-840

47 Štěrbová D, Matějičček D, Vlček J, Kubáň V. Combined microwave-assisted isolation and solid-phase purification procedures prior to the chromatographic determination of phenolic compounds in plant materials. Anal Chim Acta 2004; 513: 435-444

48 Rijo P, Fale PL, Serralheiro ML, Simoes MF, Gomes A, Reis C. Optimization of medicinal plant extraction methods and their encapsulation through extrusion technology. Measurement 2014; 58: 249-255

49 Cabana $R$, Silva LR, Valentao P, Viturro CI, Andrade PB. Effect of different extraction methodologies on the recovery of bioactive metabolites from Satureja parvifolia (Phil.) Epling (Lamiaceae). Ind Crops Prod 2013; 48: 49-56

50 Dastmalchi K, Damien Dorman HJ, Laakso I, Hiltunen R. Chemical composition and antioxidative activity of Moldavian balm (Dracocephalum moldavica L.) extracts. LWT-Food Sci Technol 2007; 40: 1655-1663

51 Bettaieb Rebey I, Kefi S, Bourgou S, Ouerghemmi I, Ksouri R, Tounsi MS, Marzouk B. Ripening stage and extraction method effects on physical properties, polyphenol composition and antioxidant activities of cumin (Cuminum cyminum L.) seeds. Plant Foods Hum Nutr 2014; 69: 358-364

52 Plánder S, Gontaru L, Blazics B, Veres K, Kéry Á, Kareth S, Simándi B. Major antioxidant constituents from Satureja hortensis L. extracts obtained with different solvents. Eur J Lipid Sci Technol 2012; 114: 772 779

53 Hadolin M, Knez Ž, Bauman D. Concentration of rosmarinic acid with supercritical fluids. Kem Ind 2003; 52: 427-432

54 Peng CH, Su JD, Chyau CC, Sung TY, Ho SS, Peng CC, Peng RY. Supercritica fluid extracts of rosemary leaves exhibit potent anti-inflammation and anti-tumor effects. Biosci Biotechnol Biochem 2007; 71: 2223-2232

55 Carvalho RN jr., Moura LS, Rosa PTV, Meireles MAA. Supercritical fluid extraction from rosemary (Rosmarinus officinalis): kinetic data, extract's global yield, composition, and antioxidant activity. J Supercrit Fluids 2005; 35: 197-204

56 Herrero M, Plaza M, Cifuentes A, Ibanez E. Green processes for the extraction of bioactives from rosemary: chemical and functional characterization via ultra-performance liquid chromatography-tandem mass spectrometry and in-vitro assays. J Chromatogr A 2010; 1217: 25122520

57 Li Y, Wang N, Zhang M, Ito Y, Zhang H, Wang Y, Guo X, Hu P. Development of a method to extract and purify target compounds from medicinal plants in a single step: online hyphenation of expanded bed adsorption chromatography and countercurrent chromatography. Anal Chem 2014; 86: 3373-3379

58 Zhu F, Asada T, Sato A, Koi Y, Nishiwaki H, Tamura H. Rosmarinic acid extract for antioxidant, antiallergic, and alpha-glucosidase inhibitory 
activities, isolated by supramolecular technique and solvent extraction from Perilla leaves. J Agric Food Chem 2014; 62: 885-892

59 Baskan S, Oztekin N, Erim FB. Determination of carnosic acid and rosmarinic acid in sage by capillary electrophoresis. Food Chem 2007; 101: 1748-1752

60 Santhiago M, Peralta RA, Neves A, Micke GA, Vieira IC. Rosmarinic acid determination using biomimetic sensor based on purple acid phosphatase mimetic. Anal Chim Acta 2008; 613: 91-97

61 Oztürk M, Duru ME, Ince B, Harmandar M, Topçu G. A new rapid spectrophotometric method to determine the rosmarinic acid level in plant extracts. Food Chem 2010; 123: 1352-1356

62 Saltas D, Pappas CS, Daferera D, Tarantilis PA, Polissiou MG. Direct determination of rosmarinic acid in Lamiaceae herbs using diffuse reflectance infrared fourier transform spectroscopy (DRIFTS) and chemometrics. J Agric Food Chem 2013; 61: 3235-3241

63 Jemia MB, Tundis R, Maggio A, Rosselli S, Senatore F, Menichini F, Bruno $M$, Kchouk ME, Loizzo MR. NMR-based quantification of rosmarinic and carnosic acids, GC-MS profile and bioactivity relevant to neurodegenerative disorders of Rosmarinus officinalis L. extracts. J Funct Foods 2013; 5: 1873-1882

64 Stehfest K, Boese M, Kerns G, Piry A, Wilhelm C. Fourier transform infrared spectroscopy as a new tool to determine rosmarinic acid in situ. J Plant Physiol 2004; 161: 151-156

65 Janicsak G, Haznagy-Radnai E, Engel R, Blunden G, Mathe I. TLC-densitometry of rosmarinic and caffeic acids in the evaluation of Lamiaceae species growing in Central Europe. JPC-J Planar Chromat - Mod TLC 2013; 26: 132-136

66 Domlur Thyagaraj V, Koshy R, Kachroo M, Mayachari AS, Sawant LP, Balasubramanium $M$. A validated RP-HPLC-UV/DAD method for simultaneous quantitative determination of rosmarinic acid and eugenol in Ocimum sanctum L. Pharm Methods 2013; 4: 1-5

67 Tian WJ, Jia J, Zhao L, Lü L, Li YY, Zhang H, Zhang GQ. HPLC-MS in simultaneous determination of 4 compounds in Fuzhengpingxiao capsule: Gentiopicroside, liquiritin, rosmarinic acid and harpagoside. Academic Journal of Second Military Medical University 2011; 32: 524-527

68 Couto RO, Conceicao EC, Chaul LT, Oliveira EMS, Alves SF, Rezende KR, Bara MTF, Paula JR. Validated HPLC-PDA method for rosmarinic acid quantification in rosemary. Latin Am J Pharm 2011; 30: 1951-1956

69 Jirovsky D, Kosina P, Myslinova M, Styskala J, Ulrichova J, Simanek V. HPLC analysis of rosmarinic acid in feed enriched with aerial parts of Prunella vulgaris and its metabolites in pig plasma using dual-channel coulometric detection. J Agric Food Chem 2007; 55: 7631-7637

70 Fecka I, Kowalczyk A, Cisowski W. Optimization of the separation of flavonoid glycosides and rosmarinic acid from Mentha piperita on HPTLC plates. JPC-J Planar Chromat - Mod TLC 2004; 17: 22-25

71 Wang MF, Juliani R, Zhang L, Wu QL, Simon JE. Analysis of flavonoids and rosmarinic acid contents in different varieties of mint by HPLC and LC-MS methods. Abstr Pap Am Chem S 2003; 226: U68-U68

72 Franzoi AC, Dupont J, Spinelli A, Vieira IC. Biosensor based on laccase and an ionic liquid for determination of rosmarinic acid in plant extracts. Talanta 2009; 77: 1322-1327

73 Kamatou GPP, Chen W, Viljoen AM. Quantification of rosmarinic acid in Salvia species indigenous to South Africa by HPTLC. JPC-J Planar Chromat - Mod TLC 2012; 25: 403-408

74 Coran SA, Mulas S, Mulinacci N. Crucial aspects of high performance thin layer chromatography quantitative validation. The case of determination of rosmarinic acid in different matrices. J Chromatogr A 2012; 1220: 156-161

75 Berhow MA, Affum AO, Gyan BA. Rosmarinic acid content in antidiabetic aqueous extract of Ocimum canum sims grown in Ghana. J Med Food 2012; 15: 611-620

76 Janicsak G, Mathe I, Miklossy-Vari V, Blunden G. Comparative studies of the rosmarinic and caffeic acid contents of Lamiaceae species. Biochem Syst Ecol 1999; 27: 733-738

77 Janicsak G, Mathe I. Parallel determination of rosmarinic and caffeic acids by TLC-densitometry. Chromatographia 1997; 46: 322-324

78 Nowakowska J. Normal and reversed-phase TLC determination of rosmarinic and valerenic acids. JPC-J Planar Chromat - Mod TLC 2004; 17 : 149-153

79 Nowakowska J, Marszalt M. Retention properties of rosmarinic and valerenic acids on an amino-modified hydrophilic layer. JPC-J Planar Chromat - Mod TLC 2003; 16: 369-373
80 Bandoniene D, Murkovic M, Venskutonis PR. Determination of rosmarinic acid in sage and borage leaves by high-performance liquid chromatography with different detection methods. J Chromatogr Sci 2005; 43: 372-376

81 Shanmuga Sundaram R, Ramanathan M, Rajesh R, Satheesh B, Saravanan D. LC-MS quantification of rosmarinic acid and ursolic acid in the Ocimum sanctum Linn. leaf extract (Holy Basil, Tulsi). J Liq Chromatogr Relat Technol 2012; 35: 634-650

82 Li X, Yu C, Sun W, Liu G, Jia J, Wang Y. Simultaneous determination of magnesium lithospermate $B$, rosmarinic acid, and lithospermic acid in beagle dog serum by liquid chromatography/tandem mass spectrometry. Rapid Commun Mass Spectrom 2004; 18: 2878-2882

83 Trute A, Nahrstedt A. Separation of rosmarinic acid enantiomers by three different chromatographic methods (HPLC, CE, GC) and the determination of rosmarinic acid in Hedera helix L. Phytochem Anal 1996; 7: 204-208

84 Exarchou V, Troganis A, Gerothanassis IP, Tsimidou M, Boskou D. Identification and quantification of caffeic and rosmarinic acid in complex plant extracts by the use of variable-temperature two-dimensional nuclear magnetic resonance spectroscopy. J Agric Food Chem 2001; 49: $2-8$

85 Soobrattee MA, Neergheen VS, Luximon-Ramma A, Aruoma OI, Bahorun $T$. Phenolics as potential antioxidant therapeutic agents: mechanism and actions. Mutat Res 2005; 579: 200-213

86 Ahmadvand H, Khosrobeigi A, Nemati L, Boshtam M, Jafari N, Hosseini RH, Pournia Y. Rosmarinic acid prevents the oxidation of low density lipoprotein (LDL) in vitro. J Biol Sci 2012; 12: 301-307

87 Fuhrman B, Volkova N, Rosenblat M, Aviram M. Lycopene synergistically inhibits LDL oxidation in combination with vitamin E, glabridin, rosmarinic acid, carnosic acid, or garlic. Antioxid Redox Signal 2000; 2: 491-506

88 Tache A, Radu GL, Litescu SC. Assessment of role of rosmarinic acid in preventing oxidative process of low density lipoproteins. Chem Pap 2012; 66: 1166-1170

89 Panya A, Kittipongpittaya K, Laguerre M, Bayrasy C, Lecomte J, Villeneuve P, McClements DJ, Decker EA. Interactions between $\alpha$-tocopherol and rosmarinic acid and its alkyl esters in emulsions: synergistic, additive, or antagonistic effect? J Agric Food Chem 2012; 60: 1032010330

90 Maheswarappa NB, Subbaiah V, Muthupalani M, Yamagani PK, Mohan K, Keshapaga UR, Asokan SV, Kalappurakkal RC. Antioxidant activity of carnosic acid and rosmarinic acid in raw and cooked ground chicken patties. J Sci Food Agric 2014; 94: 273-279

91 Sanchez-Campillo M, Gabaldon JA, Castillo J, Benavente-Garcia O, Del Bano MJ, Alcaraz M, Vicente V, Alvarez N, Lozano JA. Rosmarinic acid, a photo-protective agent against UV and other ionizing radiations. Food Chem Toxicol 2009; 47: 386-392

92 Murakami K, Haneda M, Qiao S, Naruse M, Yoshino M. Prooxidant action of rosmarinic acid: transition metal-dependent generation of reactive oxygen species. Toxicol In Vitro 2007; 21: 613-617

93 Munoz-Munoz JL, Garcia-Molina F, Ros E, Tudela J, Garcia-Canovas F, Rodriguez-Lopez JN. Prooxidant and antioxidant activities of rosmarinic acid. J Food Biochem 2013; 37: 396-408

94 Gil Ede S, Enache TA, Oliveira-Brett AM. Redox behaviour of verbascoside and rosmarinic acid. Comb Chem High Throughput Screen 2013; 16: 92-97

95 Popov AM, Osipov AN, Korepanova EA, Krivoshapko ON, Artyukov AA. [Study of antioxidant and membrane activity of rosmarinic acid using different model systems]. Biophysics (Russian Federation) 2013; 58: 607-615

96 Fadel O, El Kirat K, Morandat S. The natural antioxidant rosmarinic acid spontaneously penetrates membranes to inhibit lipid peroxidation in situ. BBA. Biomembranes 2011; 1808: 2973-2980

97 Fujimoto A, Masuda T. Antioxidation mechanism of rosmarinic acid, identification of an unstable quinone derivative by the addition of odourless thiol. Food Chem 2012; 132: 901-906

98 Wu TT, Hwang BR, Cho EJ. Scavenging effect of extract from Perilla frutescens and rosmarinic acid from free radical and lipid peroxidation. J Food Sci Nutr 2011; 16: 224-229

99 Pirker KF, Kay CW, Stolze K, Tunega D, Reichenauer T, Goodman B. Free radical generation in rosmarinic acid investigated by electron paramagnetic resonance spectroscopy. Free Radic Res 2009; 43: 47-57

100 Qiao S, Li W, Tsubouchi R, Haneda M, Murakami K, Takeuchi F, Nisimoto $Y$, Yoshino M. Rosmarinic acid inhibits the formation of reactive oxy- 
gen and nitrogen species in RAW264.7 macrophages. Free Radic Res 2005; 39: 995-1003

101 Nakamura Y, Ohto Y, Murakami A, Ohigashi H. Superoxide scavenging activity of rosmarinic acid from Perilla frutescens Britton var. acuta f. viridis. J Agric Food Chem 1998; 46: 4545-4550

102 Chen $S, F u Y, W u R$. Effects of rosmarinic acid on free radical production and lysosomal enzyme release from rat peritoneal neutrophils. Acta Pharm Sin 1999; 34: 881-885

103 Lamaison JL, Petitjean-Freytet C, Carnat A. [Medicinal Lamiaceae with antioxidant properties, a potential source of rosmarinic acid]. Pharm Acta Helv 1991; 66: 185-188

104 Lamaison JL, Petitjean-Freytet C, Duband F, Carnat AP. Rosmarinic acid content and antioxidant activity in French Lamiaceae. Fitoterapia 1991; 62: 166-171

105 Toussaint JP, Kraml M, Nell M, Smith SE, Smith FA, Steinkellner S, Schmiderer C, Vierheilig H, Novak J. Effect of Glomus mosseae on concentrations of rosmarinic and caffeic acids and essential oil compounds in basil inoculated with Fusarium oxysporum f.sp basilici. Plant Pathol 2008; 57: 1109-1116

106 Tobimatsu Y, Elumalai S, Grabber JH, Davidson CL, Pan X, Ralph J. Hydroxycinnamate conjugates as potential monolignol replacements: in vitro lignification and cell wall studies with rosmarinic acid. ChemSusChem 2012; 5: 676-686

107 Qiang Z, Ye Z, Hauck C, Murphy PA, McCoy JA, Widrlechner MP, Reddy $M B$, Hendrich $S$. Permeability of rosmarinic acid in Prunella vulgaris and ursolic acid in Salvia officinalis extracts across Caco-2 cell monolayers. J Ethnopharmacol 2011; 137: 1107-1112

108 Konishi Y, Kobayashi S. Transepithelial transport of rosmarinic acid in intestinal Caco-2 cell monolayers. Biosci Biotechnol Biochem 2005; 69: 583-591

109 Bel-Rhlid R, Crespy V, Page-Zoerkler N, Nagy K, Raab T, Hansen CE. Hydrolysis of rosmarinic acid from rosemary extract with esterases and Lactobacillus johnsonii in vitro and in a gastrointestinal model. J Agric Food Chem 2009; 57: 7700-7705

110 Falé PL, Ascensao L, Serralheiro MLM. Effect of luteolin and apigenin on rosmarinic acid bioavailability in Caco-2 cell monolayers. Food Funct 2013: 4: 426-431

111 Lin $Y Y$, Chen $P, X u X J$. Investigation of rosmarinic acid inhibition towards UDP-glucuronosyltransferases (UGTs) isoforms. Latin Am J Pharm 2013; 32: 459-461

112 Li FR, Fu YY, Jiang DH, Wu Z, Zhou YJ, Guo L, Dong ZM, Wang ZZ. Reversal effect of rosmarinic acid on multidrug resistance in SGC7901/Adr cell. J Asian Nat Prod Res 2013; 15: 276-285

113 Xu Y, Xu G, Liu L, Xu D, Liu J. Anti-invasion effect of rosmarinic acid via the extracellular signal-regulated kinase and oxidation-reduction pathway in Ls174-T cells. J Cell Biochem 2010; 111: 370-379

114 Anusuya C, Manoharan S. Antitumor initiating potential of rosmarinic acid in 7,12-dimethylbenz(a)anthracene-induced hamster buccal pouch carcinogenesis. J Environ Pathol Toxicol Oncol 2011; 30: 199211

115 Sharmila $R$, Manoharan S. Anti-tumor activity of rosmarinic acid in 7,12-dimethylbenz(a)anthracene (DMBA) induced skin carcinogenesis in Swiss albino mice. Indian J Exp Biol 2012; 50: 187-194

116 Venkatachalam K, Gunasekaran S, Jesudoss VA, Namasivayam N. The effect of rosmarinic acid on 1,2-dimethylhydrazine induced colon carcinogenesis. Exp Toxicol Pathol 2013; 65: 409-418

117 Karthikkumar V, Sivagami G, Vinothkumar R, Rajkumar D, Nalini N. Modulatory efficacy of rosmarinic acid on premalignant lesions and antioxidant status in 1,2-dimethylhydrazine induced rat colon carcinogenesis. Environ Toxicol Pharmacol 2012; 34: 949-958

118 Karmokar A, Marczylo TH, Cai H, Steward WP, Gescher AJ, Brown K. Dietary intake of rosmarinic acid by ApcMin mice, a model of colorectal carcinogenesis: levels of parent agent in the target tissue and effect on adenoma development. Mol Nutr Food Res 2012; 56: 775-783

119 Kim JH, Lee BJ, Kim JH, Yu YS, Kim MY, Kim KW. Rosmarinic acid suppresses retinal neovascularization via cell cycle arrest with increase of p21(WAF1) expression. Eur J Pharmacol 2009; 615: 150-154

120 Velpandian T, Gupta P, Ravi AK, Sharma HP, Biswas NR. Evaluation of pharmacological activities and assessment of intraocular penetration of an ayurvedic polyherbal eye drop (Itone ${ }^{\mathrm{TM}}$ ) in experimental models. BMC Complement Altern Med 2013; 13: 1-12

121 Furtado MA, Fernandes de Almeida LC, Furtado RA, Cunha WR, Tavares $D C$. Antimutagenicity of rosmarinic acid in Swiss mice evaluated by the micronucleus assay. Mutat Res - Genet Toxicol Environ Mutagen 2008; 657: 150-154
122 De Oliveira NCD, Sarmento MS, Nunes EA, Porto CM, Rosa DP, Bona SR, Rodrigues G, Marroni NP, Pereira P, Picada JN, Ferraz ABF, Thiesen FV, Da Silva J. Rosmarinic acid as a protective agent against genotoxicity of ethanol in mice. Food Chem Toxicol 2012; 50: 1208-1214

123 Wang SW, Liu XL, Yu ZY, Han QF, Cong YW. Protective effect of rosmarinic acid on $\gamma$ ray-induced micronucleus in polychromatic erythrocytes of murine bone marrow cells. J Int Pharm Res 2011; 38: 381-384

124 Ku SK, Yang EJ, Song KS, Bae JS. Rosmarinic acid down-regulates endothelial protein $C$ receptor shedding in vitro and in vivo. Food Chem Toxicol 2013; 59: 311-315

125 Yang EJ, Ku SK, Lee W, Lee S, Lee T, Song KS, Bae JS. Barrier protective effects of rosmarinic acid on HMGB1-induced inflammatory responses in vitro and in vivo. J Cell Physiol 2013; 228: 975-982

126 Englberger W, Hadding U, Etschenberg E, Graf E, Leyck S, Winkelmann J, Parnham MJ. Rosmarinic acid - A new inhibitor of complement C3convertase with antinflammatory activity. Int J Immunopharmacol 1988; 10: 729-737

127 Boonyarikpunchai $W$, Sukrong S, Towiwat P. Antinociceptive and antiinflammatory effects of rosmarinic acid isolated from Thunbergia laurifolia Lindl. Pharmacol Biochem Behav 2014; 124: 67-73

128 Youn J, Lee KH, Won J, Huh SJ, Yun HS, Cho WG, Paik DJ. Beneficial effects of rosmarinic acid on suppression of collagen induced arthritis. J Rheumatol 2003; 30: 1203-1207

129 Oh HA, Park CS, Ahn HJ, Park YS, Kim HM. Effect of Perilla frutescens var. acuta Kudo and rosmarinic acid on allergic inflammatory reactions. Exp Biol Med 2011; 236: 99-106

130 Chu X, Ci X, He J, Jiang L, Wei M, Cao Q, Guan M, Xie X, Deng X. Effects of a natural prolyl oligopeptidase inhibitor, rosmarinic acid, on lipopolysaccharide-induced acute lung injury in mice. Molecules 2012; 17: 3586-3598

131 Costa RS, Brasil Carneiro TC, Cerqueira-Lima AT, Queiroz NV, AlcantaraNeves NM, Pontes-de-Carvalho LC, Velozo EdS, Oliveira EJ, Figueiredo CA. Ocimum gratissimum Linn. and rosmarinic acid, attenuate eosinophilic airway inflammation in an experimental model of respiratory allergy to Blomia tropicalis. Int Immunopharmacol 2012; 13: 126-134

132 Jang AH, Kim TH, Kim GD, Park CS. Administration of rosmarinic acid ameliorated DNFB-induced atopic dermatitis like symptoms in NC/ Nga mice. Toxicol Lett 2010; 196: S196

133 Jang AH, Kim TH, Kim GD, Kim JE, Kim HJ, Kim SS, Jin YH, Park YS, Park CS. Rosmarinic acid attenuates 2,4-dinitrofluorobenzene-induced atopic dermatitis in NC/Nga mice. Int Immunopharmacol 2011; 11 : 1271-1277

134 Jiang WL, Chen XG, Qu GW, Yue XD, Zhu HB, Tian JW, Fu FH. Rosmarinic acid protects against experimental sepsis by inhibiting proinflammatory factor release and ameliorating hemodynamics. Shock 2009; 32: 608-613

135 Sanbongi C, Takano H, Osakabe N, Sasa N, Natsume M, Yanagisawa R, Inoue K, Kato Y, Osawa T, Yoshikawa T. Rosmarinic acid inhibits lung injury induced by diesel exhaust particles. Free Radic Biol Med 2003; 34: 1060-1069

136 Swarup V, Ghosh J, Ghosh S, Saxena A, Basu A. Antiviral and anti-inflammatory effects of rosmarinic acid in an experimental murine model of Japanese encephalitis. Antimicrob Agents Chemother 2007; 51: 3367-3370

137 Yun SY, Hur YG, Kang MA, Lee J, Ahn C, Won J. Synergistic immunosuppressive effects of rosmarinic acid and rapamycin in vitro and in vivo. Transplantation 2003; 75: 1758-1760

138 Jung DY, Kim EY, Joo SY, Jae BP, Moon C, Sa HK, Sim EY, Joh JW, Choon HK, Kwon GY, Kim SJ. Prolonged survival of islet allografts in mice treated with rosmarinic acid and anti-CD154 antibody. Exp Mol Med 2008; 40: 1-10

139 Aung HT, Nikai T, Komori Y, Nonogaki T, Niwa M, Takaya Y. Biological and pathological studies of rosmarinic acid as an inhibitor of hemorrhagic Trimeresurus flavoviridis (habu) venom. Toxins (Basel) 2010; 2: 2478-2489

140 Aung HT, Nikai T, Niwa M, Takaya Y. Rosmarinic acid in Argusia argentea inhibits snake venom-induced hemorrhage. J Nat Med 2010; 64: 482-486

141 Aung HT, Furukawa T, Nikai T, Niwa M, Takaya Y. Contribution of cinnamic acid analogues in rosmarinic acid to inhibition of snake venom induced hemorrhage. Bioorg Med Chem 2011; 19: 2392-2396

142 Dos Santos JI, Santos-Filho NA, Soares AM, Fontes MRM. Crystallization and preliminary X-ray crystallographic studies of a Lys49-phospholipase A2 homologue from Bothrops pirajai venom complexed with 
rosmarinic acid. Acta Crystallogr F Struct Biol Commun 2010; 66: 699-701

143 Dos Santos JI, Cardoso FF, Soares AM, Dal Pai Silva M, Gallacci M, Fontes $M R$. Structural and functional studies of a bothropic myotoxin complexed to rosmarinic acid: new insights into Lys49-PLA 2 inhibition. PLoS One 2011; 6: e28521

144 Ticli FK, Hage LIS, Cambraia RS, Pereira PS, Magro AJ, Fontes MRM, Stabeli RG, Giglio JR, Franca SC, Soares AM, Sampaio SV. Rosmarinic acid, a new snake venom phospholipase $\mathrm{A}(2)$ inhibitor from Cordia verbenacea (Boraginaceae): antiserum action potentiation and molecular interaction. Toxicon 2005; 46: 318-327

145 Parker-Cote JL, O'Rourke DP, Miller SN, Brewer KL, Rosenbaum MD, Meggs WJ. Trypsin and rosmarinic acid reduce the toxicity of Micrurus fulvius venom in mice. Clin Toxicol (Phila) 2014; 52: 118-120

146 Rossi F, Jullian V, Pawlowiez R, Kumar-Roine S, Haddad M, Darius HT, Gaertner-Mazouni N, Chinain M, Laurent D. Protective effect of Heliotropium foertherianum (Boraginaceae) folk remedy and its active compound, rosmarinic acid, against a Pacific ciguatoxin. J Ethnopharmacol 2012; 143: 33-40

147 Zou ZW, Xu LN, Tian JY. [Antithrombotic and antiplatelet effects of rosmarinic acid, a water-soluble component isolated from Radix Salviae Miltiorrhizae (Danshen)]. Acta Pharm Sin 1993; 28: 241-245

148 Domitrović R, Potočnjak I, Crnčević-Orlić T, Škoda M. Nephroprotective activities of rosmarinic acid against cisplatin-induced kidney injury in mice. Food Chem Toxicol 2014; 66: 321-328

149 Tian J, Jiang $W$, Hou J, Wu H. Effect of rosmarinic acid on puromycin aminonucleoside induced nephrosis in rats. Toxicol Lett 2006; 164: S228

150 Tian J, Jiang $W$. Rosmarinic acid inhibits acute renal injury and cyst formation in cisplatin-treated rat kidney. Toxicol Lett 2006; 164: S265

151 Makino T, Ono T, Muso E, Yoshida H, Honda G, Sasayama S. Inhibitory effects of rosmarinic acid on the proliferation of cultured murine mesangial cells. Nephrol Dial Transplant 2000; 15: 1140-1145

152 Jeong HJ, Choi Y, Kim MH, Kang IC, Lee JH, Park C, Park R, Kim HM. Rosmarinic acid, active component of Dansam-Eum attenuates ototoxicity of cochlear hair cells through blockage of caspase- 1 activity. PLoS One 2011; 6: e18815

153 Jiang $W L, X u Y$, Zhang SP, Hou J, Zhu HB. Effect of rosmarinic acid on experimental diabetic nephropathy. Basic Clin Pharmacol Toxicol 2012; 110: 390-395

154 Tavafi $M$, Ahmadvand $H$. Effect of rosmarinic acid on inhibition of gentamicin induced nephrotoxicity in rats. Tissue Cell 2011; 43: 392-397

155 Tavafi M, Ahmadvand H, Khalatbari A, Tamjidipoor A. Rosmarinic acid ameliorates diabetic nephropathy in uninephrectomized diabetic rats. Iran J Basic Med Sci 2011; 14: 275-283

156 Alkam T, Nitta A, Itoh A, Mizoguchi H, Tsunekawa H, Mouri A, Noda Y, Nabeshima T. Rosmarinic acid protects against memory impairments induced by acute i.c.v. injection of A beta(25-35). J Pharmacol Sci 2006; $100: 147 \mathrm{p}$

157 Karthik D, Viswanathan P, Anuradha CV. Administration of rosmarinic acid reduces cardiopathology and blood pressure through inhibition of $\mathrm{p} 22$ phox NADPH oxidase in fructose-fed hypertensive rats. J Cardiovasc Pharmacol 2011; 58: 514-521

158 Karthik D, Arunkumar E, Viswanathan P, Anuradha CV. Rosmarinic acid treatment alleviates fibrotic changes in the myocardium induced in a rat model of insulin resistance. Asian Pac J Trop Dis 2012; 2 (Suppl. 2): S920-S926

159 Sotnikova R, Okruhlicova L, Vlkovicova J, Navarova J, Gajdacova B, Pivackova L, Fialova S, Krenek P. Rosmarinic acid administration attenuates diabetes-induced vascular dysfunction of the rat aorta. J Pharm Pharmacol 2013; 65: 713-723

160 Rocha JP, Sepodes BM, Eduardo-Figueira M, Bronze R, Silva-Lima B, Mota-Filipe $H$. Rosmarinic acid reduces multiple organ failure in thermal injury and liver ischemia-reperfusion injury. FASEB J 2009; 23: 739.15

161 Yang MD, Chiang YM, Higashiyama R, Asahina K, Mann DA, Mann J, Wang CC, Tsukamoto $H$. Rosmarinic acid and baicalin epigenetically derepress peroxisomal proliferator-activated receptor gamma in hepatic stellate cells for their antifibrotic effect. Hepatology 2012; 55: $1271-1281$

162 Fialova S, Sotnikova R, Nosalova V, Drabikova K, Tekelova D, Kotalova D, Grancai $D$. Ex vivo protective effect of rosmarinic acid and Mentha $\times$ villosa water extract on tissue injury induced by ischaemia/reperfusion. Planta Med 2010; 76: 1278-1279

163 Domitrović R, Škoda M, Vasiljev Marchesi V, Cvijanović O, Pernjak Pugel E, Štefan MB. Rosmarinic acid ameliorates acute liver damage and fi- brogenesis in carbon tetrachloride-intoxicated mice. Food Chem Toxicol 2013; 51: 370-378

164 Li GS, Jiang WL, Tian JW, Qu GW, Zhu HB, Fu FH. In vitro and in vivo antifibrotic effects of rosmarinic acid on experimental liver fibrosis. Phytomedicine 2010; 17: 282-288

165 Osakabe N, Yasuda A, Natsume M, Sanbongi C, Kato Y, Osawa T, Yoshikawa T. Rosmarinic acid, a major polyphenolic component of Perilla frutescens, reduces lipopolysaccharide (LPS)-induced liver injury in $D$-galactosamine (D-GalN)-sensitized mice. Free Radic Biol Med 2002; 33: 798-806

166 Mushtaq N, Schmatz R, Pereira LB, Ahmad M, Stefanello N, Vieira JM, Abdalla F, Rodrigues MV, Baldissarelli J, Pelinson LP, Dalenogare DP, Reichert KP, Dutra EM, Mulinacci N, Innocenti M, Bellumori M, Morsch VM, Schetinger MR. Rosmarinic acid prevents lipid peroxidation and increase in acetylcholinesterase activity in brain of streptozotocin-induced diabetic rats. Cell Biochem Funct 2014; 32: 287-293

167 Luan H, Kan Z, Xu Y, Lv C, Jiang W. Rosmarinic acid protects against experimental diabetes with cerebral ischemia: relation to inflammation response. J Neuroinflammation 2013; 10: 28

168 Wang J, Xu H, Jiang H, Du X, Sun P, Xie J. Neurorescue effect of rosmarinic acid on 6-hydroxydopamine-lesioned nigral dopamine neurons in rat model of Parkinson's disease. J Mol Neurosci 2012; 47: 113-119

169 Nie H, Peng Z, Lao N, Wang H, Chen Y, Fang Z, Hou W, Gao F, Li X, Xiong L, Tan $Q$. Rosmarinic acid ameliorates PTSD-like symptoms in a rat model and promotes cell proliferation in the hippocampus. Prog Neuropsychopharmacol Biol Psychiatry 2014; 51: 16-22

170 Jin X, Liu P, Yang F, Zhang YH, Miao D. Rosmarinic acid ameliorates depressive-like behaviors in a rat model of CUS and up-regulates BDNF Levels in the hippocampus and hippocampal-derived astrocytes. Neurochem Res 2013; 38: 1828-1837

171 Tawaratsumida Y, Sunagawa M, Kaneki K, Kijima T, Yoshida Y, Yoshida $N$, Hisamitsu $T$. Effect of rosmarinic acid on social isolation stress in rats. Jpn Pharmacol Ther 2013; 41: 43-50

172 Takeda H, Tsuji M, Miyamoto J, Matsumiya T. Rosmarinic acid and caffeic acid reduce the defensive freezing behavior of mice exposed to conditioned fear stress. Psychopharmacology (Berl) 2002; 164: 233 235

173 Takeda H, Tsuji M, Miyamoto J, Matsumiya T. Caffeic acid, an active metabolite of rosmarinic acid, reduces the freezing behavior of mice exposed to conditioned fear stress. J Pharm Sci 2003; 91: 191P-191P

174 Ito N, Yabe T, Gamo Y, Nagai T, Oikawa T, Yamada H, Hanawa T. Rosmarinic acid from Perillae Herba produces an antidepressant-like effect in mice through cell proliferation in the hippocampus. Biol Pharm Bull 2008; 31: 1376-1380

175 Takeda H, Tsuji M, Matsumiya T, Kubo M. Identification of rosmarinic acid as a novel antidepressive substance in the leaves of Perilla frutescens Britton var. acuta Kudo (Perillae Herba). Nihon Shinkei Seishin Yakurigaku Zasshi 2002; 22: 15-22

176 Takeda H, Tsuji M, Inazu M, Egashira T, Matsumiya T. Rosmarinic acid and caffeic acid produce antidepressive-like effect in the forced swimming test in mice. Eur J Pharmacol 2002; 449: 261-267

177 Pereira P, Tysca D, Oliveira P, Brun LFD, Picada JN, Ardenghi P. Neurobehavioral and genotoxic aspects of rosmarinic acid. Pharmacol Res 2005; 52: 199-203

178 Park DH, Park SJ, Kim JM, Jung WY, Ryu JH. Subchronic administration of rosmarinic acid, a natural prolyl oligopeptidase inhibitor, enhances cognitive performances. Fitoterapia 2010; 81: 644-648

179 Shimojo Y, Kosaka K, Noda Y, Shimizu T, Shirasawa T. Effect of rosmarinic acid in motor dysfunction and life span in a mouse model of familial amyotrophic lateral sclerosis. J Neurosci Res 2010; 88: 896-904

180 Van Dyke T, Braswell L, Offenbacher S. Inhibition of gingivitis by topical application of ebselen and rosmarinic acid. Inflamm Res 1986; 19: 376-377

181 Ranjbar A, Khorami S, Safarabadi M, Shahmoradi A, Malekirad AA, Vakilian $K$, Mandegary A, Abdollahi M. Antioxidant activity of Iranian Echium amoenum Fisch \& C.A. Mey flower decoction in humans: A cross-sectional before/after clinical trial. Evid Based Complement Alternat Med 2006; 3: 469-473

182 Mehrabani M, Ghannadi A, Sajjadi E, Ghassemi N, Shams-Ardakani M. Toxic pyrrolizidine alkaloids of Echium amoenum Fisch. and Mey. DARU 2006; 14: 122-127

183 Rodriguez-Mateos A, Rendeiro C, Bergillos-Meca T, Tabatabaee S, George TW, Heiss C, Spencer JP. Intake and time dependence of blueberry flavonoid-induced improvements in vascular function: a randomized, controlled, double-blind, crossover intervention study with 
mechanistic insights into biological activity. Am J Clin Nutr 2013; 98 : 1179-1191

184 Li Z, Henning SM, Zhang Y, Zerlin A, Li L, Gao K, Lee RP, Karp H, Thames $G$, Bowerman S, Heber D. Antioxidant-rich spice added to hamburger meat during cooking results in reduced meat, plasma, and urine malondialdehyde concentrations. Am J Clin Nutr 2010; 91: 1180-1184

185 Viigimaa M, Abina J, Zemtsovskaya G, Tikhaze A, Konovalova G, Kumskova E, Lankin V. Malondialdehyde-modified low-density lipoproteins as biomarker for atherosclerosis. Blood Press 2010; 19: 164-168

186 Goronfolah L, Ferrie P, Schmuck M, Waserman S, Denburg J, Kott L, Keith $P$, Crawford L, Fletcher R. A double-blind, placebo-controlled, randomized, crossover trial of a mint tea high in rosmarinic acid in adults with nasal polyposis. J Allergy Clin Immunol 2009; 123: S202

187 Connelly AE, Tucker AJ, Tulk H, Catapang M, Chapman L, Sheikh N, Yurchenko S, Fletcher R, Kott LS, Duncan AM. High-rosmarinic acid spearmint tea in the management of knee osteoarthritis symptoms. J Med Food 2014; 17: 1361-1367

188 Lee J, Jung E, Koh J, Kim YS, Park D. Effect of rosmarinic acid on atopic dermatitis. J Dermatol 2008; 35: 768-771

189 Osakabe N, Takano H, Sanbongi C, Yasuda A, Yanagisawa R, Inoue KI, Yoshikawa T. Anti-inflammatory and anti-allergic effect of rosmarinic acid (RA); inhibition of seasonal allergic rhinoconjunctivitis (SAR) and its mechanism. Biofactors 2004; 21: 127-131

190 Jia JY, Lu YL, Li XC, Liu GY, Li SJ, Liu Y, Liu YM, Yu C, Wang YP. Pharmacokinetics of depside salts from Salvia miltiorrhiza in healthy Chinese volunteers: A randomized, open-label, single-dose study. Curr Ther Res Clin Exp 2010; 71: 260-271

191 Baba S, Osakabe N, Natsume M, Yasuda A, Muto Y, Hiyoshi T, Takano H, Yoshikawa T, Terao J. Absorption, metabolism, degradation and urinary excretion of rosmarinic acid after intake of Perilla frutescens extract in humans. Eur J Nutr 2005; 44: 1-9

192 Nakazawa T, Ohsawa K. Metabolites of orally administered Perilla frutescens extract in rats and humans. Biol Pharm Bull 2000; 23: 122127

193 Nakazawa T, Ohsawa K. Metabolism of rosmarinic acid in rats. J Nat Prod 1998; 61: 993-996

194 Azevedo MF, Lima CF, Fernandes-Ferreira M, Almeida MJ, Wilson JM, Pereira-Wilson $C$. Rosmarinic acid, major phenolic constituent of Greek sage herbal tea, modulates rat intestinal SGLT1 levels with effects on blood glucose. Mol Nutr Food Res 2011; 55 (Suppl. 1): S15S25

195 Debersac P, Vernevaut MF, Amiot MJ, Suschetet M, Siess MH. Effects of a water-soluble extract of rosemary and its purified component rosmarinic acid on xenobiotic-metabolizing enzymes in rat liver. Food Chem Toxicol 2001; 39: 109-117

196 Konishi Y, Hitomi Y, Yoshida M, Yoshioka E. Pharmacokinetic study of caffeic and rosmarinic acids in rats after oral administration. J Agric Food Chem 2005; 53: 4740-4746

197 Baba S, Osakabe N, Natsume M, Terao J. Orally administered rosmarinic acid is present as the conjugated and/or methylated forms in plasma, and is degraded and metabolized to conjugated forms of caffeic acid, ferulic acid and $m$-coumaric acid. Life Sci 2004; 75: 165-178

198 Ma B, Wang Y, Zhang Q Liu Y, Li J, Xu Q Ying H. Simultaneous determination of oridonin, ponicidin and rosmarinic acid from Herba Isodi Rubescentis extract by LC-MS-MS in rat plasma. J Chromatogr Sci 2013; 51: 910-918

199 Liu Y, Li X, Li Y, Wang L, Xue M. Simultaneous determination of danshensu, rosmarinic acid, cryptotanshinone, tanshinone IIA, tanshinone I and dihydrotanshinone I by liquid chromatographic-mass spectrometry and the application to pharmacokinetics in rats. J Pharm Biomed Anal 2010; 53: 698-704

$200 \mathrm{Kim}$ HJ, Kim TH, Kang KC, Pyo HB, Jeong HH. Microencapsulation of rosmarinic acid using polycaprolactone and various surfactants. Int J Cosmet Sci 2010; 32: 185-191

201 Campos DA, Madureira AR, Gomes AM, Sarmento B, Pintado MM. Optimization of the production of solid Witepsol nanoparticles loaded with rosmarinic acid. Colloids Surf B Biointerfaces 2014; 115: 109117

202 Çelik SE, Özyürek M, Tufan AN, Güçlü K, Apak R. Spectroscopic study and antioxidant properties of the inclusion complexes of rosmarinic acid with natural and derivative cyclodextrins. Spectrochim Acta A Mol Biomol Spectrosc 2011; 78: 1615-1624

203 Medronho B, Valente AJ, Costa P, Romano A. Inclusion complexes of rosmarinic acid and cyclodextrins: stoichiometry, association constants, and antioxidant potential. Colloid Polym Sci 2014; 292: 885-894
204 Panya A, Kittipongpittaya K, Laguerre Ml, Bayrasy C, Lecomte J, Villeneuve P, McClements DJ, Decker EA. Interactions between $\alpha$-tocopherol and rosmarinic acid and its alkyl esters in emulsions: synergistic, additive, or antagonistic effect? J Agric Food Chem 2012; 60: 1032010330

205 Budhiraja A, Dhingra G. Development and characterization of a novel antiacne niosomal gel of rosmarinic acid. Drug Deliv 2015; 22: 723730

206 Lee MW, Yang TP, Peng HH, Chen JW. Preparation and characterization of polygalacturonic acid/rosmarinic acid membrane crosslinked by short chain hyaluronate for preventing postoperative abdominal adhesion. Carbohydr Polym 2012; 87: 1749-1755

207 Brondani D, Zapp E, Vieira IC, Dupont J, Scheeren CW. Gold nanoparticles in an ionic liquid phase supported in a biopolymeric matrix applied in the development of a rosmarinic acid biosensor. Analyst 2011; 136: 2495-2505

208 Sarkar A, Shukla SP, Adhikari S, Mukherjee T. Synthesis, stabilisation and surface modification of gold and silver nanoparticles by rosmarinic acid and its analogues. Int J Nanotechnol 2010; 7: 1027-1037

209 Akowuah GA, Ismail Z, Norhayati I, Sadikun A. The effects of different extraction solvents of varying polarities on polyphenols of Orthosiphon stamineus and evaluation of the free radical-scavenging activity. Food Chem 2005; 93: 311-317

210 Olah NK, Radu L, Mogosan C, Hanganu D, Gocan S. Phytochemical and pharmacological studies on Orthosiphon stamineus Benth. (Lamiaceae) hydroalcoholic extracts. J Pharm Biomed Anal 2003; 33: 117123

211 Geller F, Schmidt C, Gottert M, Fronza M, Schattel V, Heinzmann B, Werz $O$, Flores EM, Merfort I, Laufer S. Identification of rosmarinic acid as the major active constituent in Cordia americana. J Ethnopharmacol 2010; 128: 561-566

212 Suwanchaikasem P, Chaichantipyuth C, Sukrong S. Antioxidant-guided isolation of rosmarinic acid, a major constituent from Thunbergia laurifolia, and its use as a bioactive marker for standardization. Chiang Mai J Sci 2014; 41: 117-127

213 Abdullah Y, Schneider B, Petersen M. Occurrence of rosmarinic acid, chlorogenic acid and rutin in Marantaceae species. Phytochem Lett 2008; 1: 199-203

214 Aquino R, Morelli S, Lauro MR, Abdo S, Saija A, Tomaino A. Phenolic constituents and antioxidant activity of an extract of Anthurium versicolor leaves. J Nat Prod 2001; 64: 1019-1023

215 Lin YL, Chang YY, Kuo YH, Shiao MS. Anti-lipid-peroxidative principles from Tournefortia sarmentosa. J Nat Prod 2002; 65: 745-747

$216 L u$ YR, Foo LY. Rosmarinic acid derivatives from Salvia officinalis. Phytochemistry 1999; 51: 91-94

217 Arda N, Goren N, Kuru A, Pengsuparp T, Pezzuto JM, Qiu SX, Cordell GA. Saniculoside N from Sanicula europaea L. J Nat Prod 1997; 60: 11701173

218 Yamamoto H, Sakakibara J, Nagatsu A, Sekiya K. Inhibitors of arachidonate lipoxygenase from defatted perilla seed. J Agric Food Chem 1998; 46: 862-865

219 Lee S, Song IH, Lee JH, Yang WY, Oh KB, Shin J. Sortase A inhibitory metabolites from the roots of Pulsatilla koreana. Bioorg Med Chem Lett 2014; $24: 44-48$

220 Furukawa M, Makino M, Ohkoshi E, Uchiyama T, Fujimoto Y. Terpenoids and phenethyl glucosides from Hyssopus cuspidatus (Labiatae). Phytochemistry 2011; 72: 2244-2252

221 Satake T, Kamiya K, Saiki Y, Hama T, Fujimoto U, Kitanaka S, Kimura Y, Uzawa J, Endang H, Umar M. Studies on the constituents of fruits of Helicteres isora L. Chem Pharm Bull 1999; 47: 1444-1447

222 Wang GC, Li T, Deng FY, Li YL, Ye WC. Five new phenolic glycosides from Hedyotis scandens. Bioorg Med Chem Lett 2013; 23: 1379-1382

223 Christ B, Kesselring $K$. Process for isolating rosmarinic acid from plants. US Patent 4354035 A; 1982

224 Achamlale S, Rezzonico B, Grignon-Dubois M. Rosmarinic acid from beach waste: Isolation and HPLC quantification in Zostera detritus from Arcachon lagoon. Food Chem 2009; 113: 878-883

225 Lembo S, Balato A, Gaudiello F, Gasparri F, Monfrecola G. Ellagic and rosmarinic acid: modulatory effect on cytokines/chemokines released by keratinocytes after ultraviolet B radiation. J Invest Dermatol 2011; 131: S106

226 Lembo S, Balato A, Di Caprio R, Cirillo T, Giannini V, Gasparri F, Monfrecola $G$. The modulatory effect of ellagic acid and rosmarinic acid on ultraviolet-B-induced cytokine/chemokine gene expression in skin keratinocyte (HaCaT) cells. Biomed Res Int 2014; 2014: 346793 
227 Moon DO, Kim MO, Lee JD, Choi YH, Kim GY. Rosmarinic acid sensitizes cell death through suppression of TNF-alpha-induced NF-kappa B activation and ROS generation in human leukemia U937 cells. Cancer Lett 2010; 288: 183-191

228 Huang N, Hauck C, Yum MY, Rizshsky L, Widrlechner MP, McCoy JA, Murphy PA, Dixon PM, Nikolau BJ, Birt DF. Rosmarinic acid in Prunella vulgaris ethanol extract inhibits lipopolysaccharide-induced prostaglandin E2 and nitric oxide in RAW 264.7 mouse macrophages. J Agric Food Chem 2009; 57: 10579-10589

229 Zdarilova A, Svobodova A, Simanek V, Ulrichova J. Prunella vulgaris extract and rosmarinic acid suppress lipopolysaccharide-induced alteration in human gingival fibroblasts. Toxicol In Vitro 2009; 23: $386-$ 392

230 Kim HK, Lee JJ, Lee JS, Park YM, Yoon TR. Rosmarinic acid down-regulates the LPS-induced production of monocyte chemoattractant protein-1 (MCP-1) and macrophage inflammatory protein-1alpha (MIP1alpha) via the MAPK pathway in bone-marrow derived dendritic cells. Mol Cells 2008; 26: 583-589

231 Scheckel KA, Degner SC, Romagnolo DF. Rosmarinic acid antagonizes activator protein-1-dependent activation of cyclooxygenase-2 expression in human cancer and nonmalignant cell lines. J Nutr 2008; 138: 2098-2105

232 Lee J, Jung E, Kim Y, Park J, Hong S, Hyun CG, Park D, Kim YS. Rosmarinic acid as a downstream inhibitor of IKK- $\beta$ in TNF- $\alpha$-induced upregulation of CCL11 and CCR3. Br J Pharmacol 2006; 148: 366-375

233 Zhang JJ, Wang YL, Feng XB, Song XD, Lu WB. Rosmarinic acid inhibits proliferation and induces apoptosis of hepatic stellate cells. Biol Pharm Bull 2011; 34: 343-348

$234 \mathrm{Xu} \mathrm{Y}$, Jiang Z, Ji G, Liu J. Inhibition of bone metastasis from breast carcinoma by rosmarinic acid. Planta Med 2010; 76: 956-962

235 Xavier CPR, Lima CF, Fernandes-Ferreira M, Pereira-Wilson C. Induction of apoptosis and inhibition of proliferation in colon cancer cells by Salvia fruticosa, Salvia officinalis and rosmarinic acid. Planta Med 2008; 74: 942-943

236 Xavier CP, Lima CF, Fernandes-Ferreira M, Pereira-Wilson C. Salvia fruticosa, Salvia officinalis, and rosmarinic acid induce apoptosis and inhibit proliferation of human colorectal cell lines: the role in MAPK/ ERK pathway. Nutr Cancer 2009; 61: 564-571

237 Hur YG, Yun YD, Won JW. Rosmarinic acid induces p56(lck)-dependent apoptosis in Jurkat and peripheral T cells via mitochondrial pathway independent from Fas/Fas ligand interaction. J Immunol 2004; 172: 79-87

238 Hur YG, Suh CH, Kim S, Won J. Rosmarinic acid induces apoptosis of activated $\mathrm{T}$ cells from rheumatoid arthritis patients via mitochondrial pathway. J Clin Immunol 2007; 27: 36-45

239 Huang SS, Zheng RL. Rosmarinic acid inhibits angiogenesis and its mechanism of action in vitro. Cancer Lett 2006; 239: 271-280

240 Kolettas E, Thomas C, Leneti E, Skoufos I, Mbatsi C, Sisoula C, Manos G, Evangelou A. Rosmarinic acid failed to suppress hydrogen peroxidemediated apoptosis but induced apoptosis of Jurkat cells which was suppressed by Bcl-2. Mol Cell Biochem 2006; 285: 111-120

241 Vattem DA, Jang HD, Levin R, Shetty K. Synergism of cranberry phenolics with ellagic acid and rosmarinic acid for antimutagenic and DNA protection functions. J Food Biochem 2006; 30: 98-116

242 Yoshida M, Fuchigami M, Nagao T, Okabe H, Matsunaga K, Takata J, Karube Y, Tsuchihashi R, Kinjo J, Mihashi K, Fujioka T. Antiproliferative constituents from Umbelliferae plants VII. Active triterpenes and rosmarinic acid from Centella asiatica. Biol Pharm Bull 2005; 28: 173175

243 Alcaraz M, Alcaraz-Saura M, Achel DG, Olivares A, Antonio Lopez-Morata J, Castillo J. Radiosensitizing effect of rosmarinic acid in metastatic melanoma B16F10 cells. Anticancer Res 2014; 34: 1913-1921

244 Braidy N, Matin A, Rossi F, Chinain M, Laurent D, Guillemin GJ. Neuroprotective effects of rosmarinic acid on ciguatoxin in primary human neurons. Neurotox Res 2014; 25: 226-234

245 Costa P, Sarmento B, Goncalves S, Romano A. Protective effects of Lavandula viridis L'Her extracts and rosmarinic acid against $\mathrm{H} 2 \mathrm{O} 2$-induced oxidative damage in A172 human astrocyte cell line. Ind Crops Prod 2013; 50: 361-365

246 Jeon YJ, Song KS, Han HJ, Park SH, Chang W, Lee MY. Rosmarinic acid inhibits chemical hypoxia-induced cytotoxicity in primary cultured rat hepatocytes. Arch Pharm Res 2014; 37: 907-915

$247 \mathrm{Kim}$ SJ, Um JY, Kim SH, Hong SH. Protective effect of rosmarinic acid is through regulation of inflammatory cytokine in cadmium-induced ototoxicity. Am J Chin Med 2013; 41: 391-404
248 Jeong HJ, Choi Y, Kim MH, Kang IC, Lee JH, Park C, Park R, Kim HM. Rosmarinic acid, active component of Dansam-Eum attenuates ototoxicity of cochlear hair cells through blockage of caspase- 1 activity. PLoS One 2011 ; 6: e18815

249 Yang SY, Hong CO, Lee GP, Kim CT, Lee KW. The hepatoprotection of caffeic acid and rosmarinic acid, major compounds of Perilla frutescens, against t-BHP-induced oxidative liver damage. Food Chem Toxicol 2013; 55: 92-99

$250 \mathrm{Du}$ T, Li L, Song N, Xie J, Jiang H. Rosmarinic acid antagonized 1-methyl-4-phenylpyridinium (MPP+)-induced neurotoxicity in MES23.5 dopaminergic cells. Int J Toxicol 2010; 29: 625-633

251 Ren P, Jiang H, Li R, Wang J, Song N, Xu HM, Xie JX. Rosmarinic acid inhibits 6-OHDA-induced neurotoxicity by anti-oxidation in MES23.5 cells. J Mol Neurosci 2009; 39: 220-225

252 Furtado RA, De Araújo FRR, Resende FA, Cunha WR, Tavares DC. Protective effect of rosmarinic acid on V79 cells evaluated by the micronucleus and comet assays. J Appl Toxicol 2010; 30: 254-259

253 Fallarini S, Miglio G, Paoletti T, Minassi A, Amoruso A, Bardelli C, Brunelleschi $S$, Lombardi G. Clovamide and rosmarinic acid induce neuroprotective effects in in vitro models of neuronal death. Br J Pharmacol 2009; 157: 1072-1084

254 Lee HJ, Cho HS, Park E, Kim S, Lee SY, Kim CS, Kim DK, Kim SJ, Chun HS. Rosmarinic acid protects human dopaminergic neuronal cells against hydrogen peroxide-induced apoptosis. Toxicology 2008; 250: 109115

255 Salimei PS, Marfe G, Di Renzo L, Di Stefano C, Giganti MG, Filomeni G, Ciriolo $M R$. The interference of rosmarinic acid in the DNA fragmentation induced by osmotic shock. Front Biosci 2007; 12: 1308-1317

256 Iuvone T, De Filippis D, Esposito G, D'Amico A, Izzo AA. The spice sage and its active ingredient rosmarinic acid protect PC12 cells from amyloid-beta peptide-induced neurotoxicity. J Pharmacol Exp Ther 2006; 317: 1143-1149

257 Psotova J, Svobodova A, Kolarova H, Walterova D. Photoprotective properties of Prunella vulgaris and rosmarinic acid on human keratinocytes. J Photochem Photobiol B 2006; 84: 167-174

258 Yan T, Yan FX, Zhou JM, Zhu BY, Gong YZ, Xu CX, Chi XL, Zhang HQ. Effect of rosmarinic acid against PC12 cell injures induced by glutamate. Chinese Pharmacological Bulletin 2006; 22: 955-959

259 Gao LP, Wei HL, Zhao HS, Xiao SY, Zheng RL. Antiapoptotic and antioxidant effects of rosmarinic acid in astrocytes. Pharmazie 2005; 60: 62-65

260 Kim DS, Kim HR, Woo ER, Hong ST, Chae HJ, Chae SW. Inhibitory effects of rosmarinic acid on adriamycin-induced apoptosis in H9c2 cardiac muscle cells by inhibiting reactive oxygen species and the activations of c-Jun N-terminal kinase and extracellular signal-regulated kinase. Biochem Pharmacol 2005; 70: 1066-1078

261 Chlopcikova S, Psotova A, Miketova P, Sousek J, Lichnovsky V, Simanek V. Chemoprotective effect of plant phenolics against anthracycline-induced toxicity on rat cardiomyocytes part II. Caffeic, chlorogenic and rosmarinic acids. Phytother Res 2004; 18: 408-413

262 Renzulli C, Galvano F, Pierdomenico L, Speroni E, Guerra MC. Effects of rosmarinic acid against aflatoxin B1 and ochratoxin-A-induced cell damage in a human hepatoma cell line (Hep G2). J Appl Toxicol 2004: 24: 289-296

263 Lee HJ, Jeong YI, Lee TH, Jung ID, Lee JS, Lee CM, Kim JI, Joo H, Lee JD, Park YM. Rosmarinic acid inhibits indoleamine 2,3-dioxygenase expression in murine dendritic cells. Biochem Pharmacol 2007; 73: 1412 1421

264 Ahn SC, Oh WK, Kim BY, Kang DO, Kim MS, Heo GY, Ahn JS. Inhibitory effects of rosmarinic acid on Lck SH2 domain binding to a synthetic phosphopeptide. Planta Med 2003; 69: 642-646

265 Kang SH, Park SH, Shim HS, Lee KH. Non-phosphopeptide inhibitor for Lck SH2 domain: Solid-phase synthesis and structure activity relationship of rosmarinic acid analogs. Bull Korean Chem Soc 2003; 24: 664-666

266 Won J, Hur YG, Hur EM, Park SH, Kang MA, Choi Y, Park C, Lee KH, Yun Y. Rosmarinic acid inhibits TCR-induced T cell activation and proliferation in an Lck-dependent manner. Eur J Immunol 2003; 33: 870-879

267 Kang MA, Yun SY, Won JW. Rosmarinic acid inhibits Ca2+-dependent pathways of T-cell antigen receptor-mediated signaling by inhibiting the PLC-gamma 1 and Itk activity. Blood 2003; 101: 3534-3542

268 Sahu A, Rawal N, Pangburn MK. Inhibition of complement by covalent attachment of rosmarinic acid to activated C3b. Biochem Pharmacol 1999; 57: 1439-1446 
269 Peake PW, Pussell BA, Martyn P, Timmermans V, Charlesworth JA. The inhibitory effect of rosmarinic acid on complement involves the C5 convertase. Int J Immunopharmacol 1991; 13: 853-857

270 Abedini A, Roumy V, Mahieux S, Biabiany M, Standaert-Vitse A, Rivière C, Sahpaz S, Bailleul F, Neut C, Hennebelle T. Rosmarinic acid and its methyl ester as antimicrobial components of the hydromethanolic extract of Hyptis atrorubens Poit. (Lamiaceae). Evid Based Complement Alternat Med 2013; 2013: 604536

271 Airoldi C, Sironi E, Dias C, Marcelo F, Martins A, Rauter AP, Nicotra F, Jimenez-Barbero J. Natural compounds against Alzheimer's disease: molecular recognition of a beta 1-42 peptide by Salvia sclareoides extract and its major component, rosmarinic acid, as investigated by NMR. Chem Asian J 2013; 8: 596-602

272 Marcelo F, Dias C, Martins A, Madeira PJ, Jorge T, Helena Florencio M, Javier Canada F, Cabrita EJ, Jimenez-Barbero J, Rauter AP. Molecular recognition of rosmarinic acid from Salvia sclareoides extracts by acetylcholinesterase: a new binding site detected by NMR spectroscopy. Chem Eur J 2013; 19: 6641-6649

273 Yin G, Li YM, Wei W, Jiang SH, Zhu DY, Du WH. Interactions of acetylcholinesterase with salvianolic acid B and rosmarinic acid from Salvia miltiorhiza water extract investigated by NMR relaxation rate. Chin Chem Lett 2008; 19: 747-751

274 Slobodníková L, Fialová S, Hupková H, Grančai D. Rosmarinic acid interaction with planktonic and biofilm Staphylococcus aureus. Nat Prod Commun 2013; 8: 1747-1750

275 Lin L, Dong Y, Zhao H, Wen L, Yang B, Zhao M. Comparative evaluation of rosmarinic acid, methyl rosmarinate and pedalitin isolated from Rabdosia serra (MAXIM.) HARA as inhibitors of tyrosinase and alpha-glucosidase. Food Chem 2011; 129: 884-889
276 Murata T, Miyase T, Yoshizaki F. Hyaluronidase inhibitory rosmarinic acid derivatives from Meehania urticifolia. Chem Pharm Bull (Tokyo) 2011; 59: 88-95

277 Ippoushi K, Yamaguchi Y, Itou H, Azuma K, Higashio H. Evaluation of inhibitory effects of vegetables and herbs on hyaluronidase and identification of rosmarinic acid as a hyaluronidase inhibitor in lemon balm (Melissa officinalis L.). Food Sci Technol Res 2000; 6: 74-77

278 Fale PL, Borges C, Amorim Madeira PJ, Ascensao L, Araujo MEM, Florencio $M H$, Serralheiro MLM. Rosmarinic acid, scutellarein 4'-methyl ether 7-O-glucuronide and (16S)-coleon E are the main compounds responsible for the antiacetylcholinesterase and antioxidant activity in herbal tea of Plectranthus barbatus ("falso boldo"). Food Chem 2009; 114: 798-805

279 Fale PLV, Araujo MEM, Ascensao L, Serralheiro MLM. Acetylcholinesterase inhibition by rosmarinic acid from Plectranthus (Lamiaceae) species. Planta Med 2008; 74: 960

280 Lee J, Kim YS, Park D. Rosmarinic acid induces melanogenesis through protein kinase A activation signaling. Biochem Pharmacol 2007; 74: 960-968

281 Kang HS, Kim HR, Byun DS, Park HJ, Cho JS. Rosmarinic acid as a tyrosinase inhibitors from Salvia miltiorrhiza. Nat Prod Sci 2004; 10: 80-84

282 McCue PP, Shetty K. Inhibitory effects of rosmarinic acid extracts on porcine pancreatic amylase in vitro. Asia Pac J Clin Nutr 2004; 13 : 101-106

283 Simpol LR, Otsuka H, Ohtani K, Kasai R, Yamasaki K. Nitrile glucosides and rosmarinic acid, the histamine inhibitor from Ehretia philippinensis. Phytochemistry 1994; 36: 91-95 